\title{
Weak and Compact Radio Emission in Early High-mass Star-forming Regions. II. The Nature of the Radio Sources
}

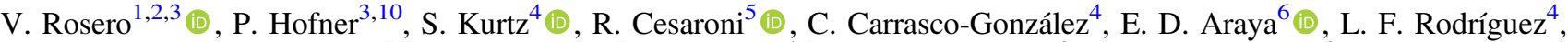 \\ K. M. Menten ${ }^{7}$ (i) , F. Wyrowski ${ }^{7}$, L. Loinard ${ }^{4,7}$ (i) , S. P. Ellingsen ${ }^{8}(1)$, and S. Molinari ${ }^{9}$ (i) \\ ${ }^{1}$ National Radio Astronomy Observatory, 1003 Lopezville Rd., Socorro, NM 87801, USA; vrosero@nrao.edu \\ ${ }^{2}$ Department of Astronomy, University of Virginia, Charlottesville, VA 22904, USA \\ ${ }^{3}$ Physics Department, New Mexico Tech, 801 Leroy Pl., Socorro, NM 87801, USA \\ ${ }^{4}$ Instituto de Radioastronomía y Astrofísica, Universidad Nacional Autónoma de México, Morelia 58090, México \\ 5 INAF, Osservatorio Astrofisico di Arcetri, Largo E. Fermi 5, I-50125 Firenze, Italy \\ ${ }^{6}$ Physics Department, Western Illinois University, 1 University Circle, Macomb, IL 61455, USA \\ ${ }_{7}$ Max-Planck-Institute für Radioastronomie, Auf dem Hügel 69, D-53121 Bonn, Germany \\ ${ }^{8}$ School of Physical Sciences, University of Tasmania, Private Bag 37, Hobart, Tasmania 7001, Australia \\ ${ }^{9}$ INAF-Istituto di Astrofisica e Planetologia Spaziale, Via Fosso del Cavaliere 100, I-00133 Roma, Italy \\ Received 2019 January 25; revised 2019 May 10; accepted 2019 May 21; published 2019 July 30
}

\begin{abstract}
In this study we analyze 70 radio continuum sources that are associated with dust clumps and which are considered to be candidates for the earliest stages of high-mass star formation. The detection of these sources was reported by Rosero et al., who found most of them to show weak $(<1 \mathrm{mJy})$ and compact $(<0$ "' 6$)$ radio emission. Herein, we used the observed parameters of these sources to investigate the origin of the radio continuum emission. We found that at least $\sim 30 \%$ of these radio detections are most likely to be ionized jets associated with high-mass protostars. However, for the most compact sources, we cannot discard the scenario that they represent pressure-confined H II regions. This result is highly relevant for recent theoretical models that are based on core accretion, which predict the first stages of ionization from high-mass stars to be in the form of jets. Additionally, we found that properties such as the radio luminosity as a function of the bolometric luminosity of ionized jets from low and high-mass stars are extremely well-correlated. Our data improve upon previous studies by providing further evidence of a common origin for jets independently of luminosity.
\end{abstract}

Key words: ISM: jets and outflows - stars: formation - HII regions - techniques: interferometric

Supporting material: data behind figures, figure set, machine-readable table

\section{Introduction}

Young high-mass stars $\left(M \geqslant 8 \quad M_{\odot}\right)$ probed in cm-wavelength interferometric studies typically appear as fairly bright (flux densities of $\sim$ few mJy to Jy) regions of ionized gas that are classified according to their size and emission measure, such as compact, ultracompact (UC), and hypercompact (HC) H II regions (e.g., Kurtz 2005). It is generally thought that once nuclear burning has begun, the star produces enough UV radiation to photoionize the surrounding gas. However, theories of the earliest stages remain poorly constrained by observations, mainly due to the characteristics of the regions where they are born, which are highly dust-obscured, distant ( $\gtrsim 1 \mathrm{kpc})$ regions that undergo rapid evolution and they reach the zeroage main sequence (ZAMS) while still heavily accreting. In fact, an evolutionary sequence for high-mass stars has not yet been established (e.g., Sánchez-Monge et al. 2013a; Tan et al. 2014), although significant progress has been achieved on both observational and theoretical fronts (e.g., Motte et al. 2018). The identification and study of objects in the early stages of their evolution will help us to discriminate among proposed mechanisms for their formation. The two main scenarios are core accretion (i.e., scaled-up version of low-mass star formation) and competitive accretion (i.e., in which stars in a cluster attract each other while they accrete from a shared reservoir of gas; see Tan et al. 2014). The low-mass star

\footnotetext{
${ }^{10}$ Adjunct Astronomer at the National Radio Astronomy Observatory, 1003 Lopezville Road, Socorro, NM 87801, USA.
}

formation process is modeled by accretion via a circumstellar disk and a collimated jet/outflow that removes angular momentum and allows accretion to proceed (e.g., Shu et al. 1988). The jet/outflow system is powered magnetohydrodynamically by rotating magnetic fields coupled to either the disk (disk winds: e.g., Konigl \& Pudritz 2000) and/or the protostar (X-winds: e.g., Shu et al. 1987). Additionally, protostellar collisions have been proposed as an alternative mechanism for the formation of high-mass stars (Bonnell et al. 1998; Bally \& Zinnecker 2005).

Massive molecular outflows are a common phenomenon in high-mass star-forming regions (e.g., Shepherd \& Churchwell 1996; Beuther et al. 2002b); hence, accretion disks and ionized jets similar to those found toward low-mass protostars are also expected. In addition, several surveys toward high-mass starforming regions in the NIR spectral lines of $\mathrm{H}_{2}$ have detected a large number of molecular jets (e.g., Navarete et al. 2015; Wolf-Chase et al. 2017). However, the current sample of known high-mass protostars associated with disks (see review by Beltrán \& de Wit 2016) and collimated jets (e.g., Marti et al. 1995; Martí et al. 1998; Curiel et al. 2006; Rodríguez et al. 2008) is inadequate to draw conclusions about the entire population. The detection of sources at the onset of high-mass star formation and the measurement of their physical properties is essential to test theoretical models of high-mass star formation (e.g., Tan et al. 2014). Furthermore, the most sensitive instruments are necessary if we wish to place 
significant constraints on the occurrence rate and parameters of these detections.

In Rosero et al. (2016, hereafter Paper I) we described our high-sensitivity $\left(\sim 3-10 \mu \mathrm{Jy}\right.$ beam $\left.^{-1}\right)$ continuum survey, which aimed to identify candidates in early evolutionary phases of high-mass star formation and to study their centimeter continuum emission. We observed 58 high-mass star-forming region candidates using the Karl G. Jansky Very Large Array $(\mathrm{VLA})^{11}$ at 1.3 and $6 \mathrm{~cm}$ wavelengths, and at an angular resolution $<0$ "' 6 . The 58 targets were grouped into three categories based on their mid and far-IR luminosity, as well as the temperature of the cores: 25 hot molecular cores (HMCs), 15 cold molecular cores with mid-IR point source association (CMC-IRs), and 18 cold molecular cores (CMCs) devoid of IR point source associations. The cores in our sample cover a wide range of parameters, including bolometric luminosity and distance. Although they have similar masses and densities, the latter two types of cores-which are mainly found within infrared dark clouds (IRDCs) - have lower temperatures $(T \sim 10-20 \mathrm{~K})$ than HMCs $(T>50 \mathrm{~K}$; depending on the probe and scale). In Paper I we reported detection rates of $1 / 18$ (6\%) CMCs, 8/15 (53\%) CMC-IRs and 25/25 (100\%) HMCs. In several cases, we detected multiple sources within a region, which resulted in a total detection of 70 radio sources associated with $1.2 \mathrm{~mm}$ dust clumps. The $100 \%$ detection rate of centimeter emission in the HMCs is a higher fraction than previously reported. This suggests that radio continuum may be present, albeit weak, in all HMCs, although in many cases it is only detectable with the superior sensitivity now available with the upgraded VLA. Our results provide further evidence for an evolutionary sequence in the formation of high-mass stars, from very early stage cold cores (i.e., CMCs) to relatively more evolved ones (i.e., HMCs).

A number of physical processes can cause centimeter continuum emission associated with high-mass star-forming regions (see Rodríguez et al. 2012 and Sánchez-Monge et al. $2013 \mathrm{~b}$ for summaries of thermal and non-thermal emission detected at centimeter wavelengths from YSOs). Recently, Tanaka et al. (2016, hereafter TTZ16) developed a model to predict the radio emission from high-mass stars forming via core accretion. The TTZ16 model predicts that during the first stages of ionization, the $\mathrm{H}$ II region is initially confined to the vertical (or outflow) axis and produces free-free emission with similar features and parameters as observed toward ionized jets. Ionized jets are detected as weak and compact centimeter continuum sources. At subarcsecond resolutions, they usually show a string-like morphology, which is often aligned with a large-scale molecular outflow of size of up to a few parsecs. Ionized jets trace outflows on smaller scales, providing the location of the driving protostar, that otherwise are deeply embedded in the natal clump and generally remain undetected at other wavelengths due to the high extinction in the region (Anglada et al. 1998). However, less extincted sources may have molecular jet counterparts that are visible in $\mathrm{H}_{2}$ line emission from shocked gas. These sources are also called "thermal radio jets," due to their characteristic rising spectrum that is consistent with free-free radiation from ionized gas. The ionization mechanism of these jets has been proposed to be shock-induced ionization when the wind from the central

\footnotetext{
11 The National Radio Astronomy Observatory is a facility of the National Science Foundation operated under cooperative agreement by Associated Universities, Inc.
}

protostar ionizes itself through shocks due to variations in velocity of the flow or variations of the mass loss rate (Curiel et al. 1987; Curiel et al. 1989). Unlike the simple model of a uniform electron density $\mathrm{H}$ II region, ionized jets and winds have a radial density gradient and are thus partially optically thick. Reynolds (1986) discussed the behavior of collimated jets and the dependency of their physical parameters (such as temperature, velocity, density and ionization fraction) on morphology, independently of the mechanism of ionization, and showed that the spectral index of a partially ionized jet ranges between $-0.1 \leqslant \alpha \leqslant 1.1$.

The detection of ionized jets toward high-mass stars at their early stages, as predicted by TTZ16, can help us to distinguish between accretion scenarios (highly organized outflows are expected from core accretion but not from competitive accretion scenarios; Tan et al. 2016), and ultimately will give us insight into the accretion disks around high-mass stars. Several systematic studies searching for ionized jets have been reported in the literature. Guzmán et al. (2012), from a sample of 33 IR luminous objects, detected two ionized jets using the Australia Telescope Compact Array (ATCA) with a $4 \sigma$ detection limit and an image $\mathrm{rms}(\sigma)$ of $\sim 0.1-0.2 \mathrm{mJy}$ beam $^{-1}$ at 4.8 and $8.6 \mathrm{GHz}$. Moscadelli et al. (2016) observed 11 high-mass YSOs using the Jansky VLA and detected five collimated ionized jets and six ionized wind candidates with a $3 \sigma$ detection limit and an $\mathrm{rms} \sim 11 \mu \mathrm{Jy}_{\text {beam }^{-1}}$ at $\sim 6.2 \mathrm{GHz}$. Purser et al. (2016) observed 49 high-mass YSOs using the ATCA and detected 16 ionized jets and 12 jet candidates with a $3 \sigma$ detection limit and an $\mathrm{rms} \sim 17 \mu \mathrm{Jy}_{\text {beam }^{-1}}$ at $\sim 5.5 \mathrm{GHz}$. Additionally, the Protostellar Outflow at the EarliesT Stage survey is undertaking a search of radio-jets using the VLA with an angular resolution of $\sim 0$. 1 and an image $\mathrm{rms}$ of $\sim 10 \mu \mathrm{Jy}$ beam $^{-1}$ (Sanna et al. 2018, 2019b). In our radio continuum Jansky VLA survey, we observed 58 high-mass star-forming regions and detected 70 radio sources with a $5 \sigma$ detection limit and an $\mathrm{rms} \sim 5 \mu \mathrm{Jy}_{\text {beam }}{ }^{-1}$ at $\sim 6 \mathrm{GHz}$ (Paper I). Anglada et al. (2018) is a recent comprehensive review of ionized jets in starforming regions.

The main goal of this paper is to investigate the nature of the 70 detected radio sources that we reported in Paper I. The observations along with the complete list of targets, coordinates, radio detections and derived observational parameters are presented in Paper I. In Section 2 we examine several scenarios to explain the origin of the ionized gas emission and we study the physical properties of the detected sources. Section 3 contains a discussion of the viability of the different scenarios. We summarize our findings in Section 4. Additionally, Appendix A gives the bolometric luminosity estimates for these high-mass star-forming regions using Herschel/Hi-GAL data and Appendix $B$ studies the momentum rate of ionized jets.

\section{Models Considered for the Radio Emission}

\subsection{Low-mass Young Stellar Objects}

The main goal of the high-sensitivity continuum survey that we presented in Paper I was to detect radio emission from highmass protostars. However, a variety of sources could also appear as radio detections in our images. In Paper I we considered contamination by extragalactic radio sources, and found that only a small number of extragalactic sources are expected to be observed within the typical dust clump size of 


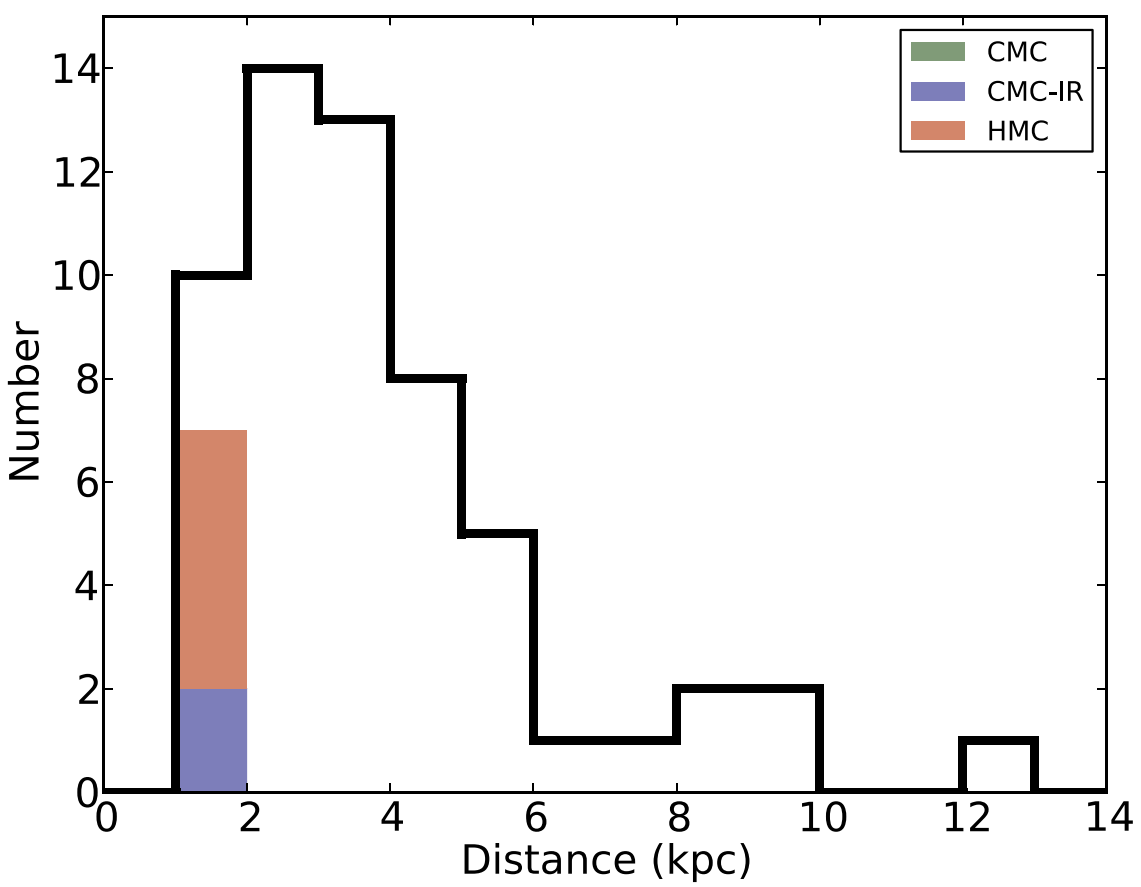

Figure 1. The distance distribution of our targets is shown as a black line. The color histogram shows the number of targets where we expect the detection of T-Tauri stars which are as bright as J162749.85-242540.5 in Ophiuchus. This is the case for five HMCs and two CMC-IRs.

$\sim 30^{\prime \prime}$ (eight and two sources in the 6 and $1.3 \mathrm{~cm}$ bands, respectively, for the entire sample). A more likely source of contamination would be the presence of low-mass YSOs, which are expected to be present in regions of high-mass star formation (e.g., Rivilla et al. 2013). We are thus interested in identifying possible low-mass class 0 -class III YSOs that could have been detected in our survey toward high-mass starforming regions.

A large sample of low-mass YSOs has been observed with the VLA at 4.5 and $7.5 \mathrm{GHz}$ as part of the Gould Belt survey (i.e., Ophiuchus at a distance of 120 pc: Dzib et al. 2013; Orion at 414 pc: Kounkel et al. 2014; Serpens at 415 pc: Ortiz-León et al. 2015; Taurus-Auriga at 140 pc: Dzib et al. 2015, and Perseus at 235 pc: Pech et al. 2016). The brightest low-mass YSO in the entire Gould Belt survey (excluding the Orion region) was found in the Ophiuchus region (source J162749.85-242540.5, a class III YSO, i.e., weak-lined T-Tauri star, with $S_{7.5 \mathrm{GHz}}=8.51 \mathrm{mJy}$, Dzib et al. 2013). To determine whether such an object would have been detected in our survey, we scaled its flux density to the assumed distance ${ }^{12}$ of each of our targets, and compared its scaled flux density to our adopted detection limit of $\geqslant 5$ times the image rms at $7.4 \mathrm{GHz}$ for each of our regions. We found that such a YSO would not be detected in any of our targets located at distances beyond $2 \mathrm{kpc}$. Since the majority of our targets exceed this distance (see Figure 1), we conclude that for most of our observed regions the detected radio sources are not low-mass YSOs.

There are 10 regions in our survey that are located at distances $\leqslant 2 \mathrm{kpc}$. However, given the $7.4 \mathrm{GHz}$ image rms for these regions, only in seven of them would we have detected the brightest lowmass YSO of Ophiuchus. These regions are five HMCs: 18517 $+0437, \quad 20126+4104, \quad 20293+3952, \quad 20343+4129, \quad$ G34.43 $+00.24 \mathrm{~mm} 1$, and two CMC-IRs: LDN1657A-3 and UYSO1.

\footnotetext{
${ }^{12}$ Distances were taken from the literature and are listed in Table 4. Most distances are kinematic, only a few regions have trigonometric parallax measurements.
}

In these seven regions, we detected a total of 13 radio sources within the FWHM of the mm clumps: 10 toward HMCs and three toward CMC-IRs. That some of these sources are possibly lowmass YSOs can be seen in the case of IRAS 20126+4104. Besides the well-studied high-mass protostars associated with radio sources $20126+4104 \mathrm{~A}$ and $20126+4104 \mathrm{~B}$, the radio source G78.121+3.632 in this region (see Paper I, Table 4) corresponds to the source I20var, which was discussed by Hofner et al. (2007). This is a highly variable radio source and has observational properties consistent with a flaring T-Tauri star. In the same region, we have also detected a new object of similar characteristics. Radio source $20126+4104 \mathrm{C}$ was detected for the first time in our survey, although several high-sensitivity observations of this region have been made in the past (Hofner et al. 2007).

Hence, $20126+4104 \mathrm{C}$ is clearly variable in the radio regime and is a candidate for a low-mass pre-main sequence star. Additionally, the radio source LDN1657A-3 A, which has a negative spectral index $(\alpha=-1.2)$, is also a candidate for a variable radio source, where the emission is probably caused by non-thermal processes on the surface of a T-Tauri star. While the observational properties of these sources are consistent with low-mass YSOs, we note that alternative explanations are possible (e.g., Cesaroni et al. 2018).

In summary, while some degree of contamination by lowmass YSOs probably exists in our survey for the nearest sources, for the majority of our targets the detected radio sources are very likely not contaminated by emission from lowmass YSOs.

\subsection{H II Regions}

In Paper I, we reported the detection of 70 radio continuum sources associated with three different types of $\mathrm{mm}$ clumps and we calculated their 5-25 GHz spectral index $(\alpha)$ using powerlaw fits of the form $S_{\nu} \propto \nu^{\alpha}$. The spectral index values and the fits to the data for all the radio detections are reported in Paper I 
Table 1

Extended Sources: Parameters from Radio Continuum

\begin{tabular}{|c|c|c|c|c|c|c|c|c|c|}
\hline Region & $\begin{array}{l}\text { Radio } \\
\text { Source }\end{array}$ & $\begin{array}{c}\nu \\
(\mathrm{GHz})\end{array}$ & $\begin{array}{c}S_{\nu} \\
(\mu \mathrm{Jy})\end{array}$ & $\begin{array}{c}\Delta s \\
(\mathrm{pc})\end{array}$ & $\begin{array}{c}\mathrm{EM} / 10^{5} \\
\left(\mathrm{pc} \mathrm{cm}{ }^{-6}\right)\end{array}$ & $\begin{array}{l}n_{e} / 10^{3} \\
\left(\mathrm{~cm}^{-3}\right)\end{array}$ & $\begin{array}{c}U \\
\left(\mathrm{pc} \mathrm{cm}^{-2}\right)\end{array}$ & $\begin{array}{c}\log N_{\mathrm{Ly}}^{\prime} \\
\left(\mathrm{s}^{-2}\right)\end{array}$ & $\begin{array}{c}\text { Spectral } \\
\text { Type }^{\mathrm{a}}\end{array}$ \\
\hline $18470-0044$ & $\mathrm{C}$ & 25.5 & 3490 & 0.085 & 8.5 & 3.2 & 9.2 & 46.4 & B0.5 \\
\hline $18521+0134$ & B & 25.5 & 378 & 0.057 & 2.5 & 2.1 & 4.7 & 45.5 & B1 \\
\hline $19035+0641$ & B & 25.5 & 2270 & 0.009 & 39.6 & 21.1 & 3.4 & 45.1 & B1 \\
\hline $20293+3952$ & $\mathrm{C}^{\mathrm{b}}$ & 25.5 & 1560 & 0.019 & 1.9 & 3.2 & 2.1 & 44.4 & B2 \\
\hline $20343+4129$ & A & 25.5 & 881 & 0.005 & 17.5 & 18.5 & 1.8 & 44.3 & B2 \\
\hline
\end{tabular}

Notes.

${ }^{\mathrm{a}}$ Using the tabulation in Panagia (1973).

b Includes radio source 20293+3952 B (see Figure 2 in Paper I).

in Table 4 (electronic version) and in Figure 4, respectively. The range of spectral indices was -1.2 to 1.8 (see Figure 5 in Paper I). Based on their radio spectra, we classify these sources as flat spectral index $(-0.25<\alpha<0.2)$, positive spectral index $(\alpha \geqslant 0.2)$, and negative spectral index $(\alpha<-0.25)$. Thus, we have 10 sources with flat, 44 sources with positive, and 9 sources with negative spectral index. For the remaining seven, there is not a clear estimate of the spectral index.

The radio sources have a variety of morphologies. Excluding the sources without spectral index information, there are six extended sources, eight sources with elongated structures, and the majority of sources (49) are compact with respect to our synthesized beam. In this section, we consider whether a family of $\mathrm{H}$ II region models can explain sources with a flat and positive spectral index.

\subsubsection{Extended Sources}

Among the sources detected in our survey associated with $1.2 \mathrm{~mm}$ dust emission, there are six sources that are clearly extended at $\mathrm{cm}$ wavelengths with respect to the $\sim 0$ !" 4 resolution of the maps, hence they are candidates for $\mathrm{H}$ II region; i.e., photoionized gas. These sources are relatively bright $\left(S_{25.5 \mathrm{GHz}} \approx 1 \mathrm{mJy}\right)$ and are found mostly toward HMCs. Moreover, they generally show a flat spectral index, which is indicative of optically thin free-free emission. For five of these sources, we calculate the physical properties from the $25.5 \mathrm{GHz}$ continuum flux using the formulae from Kurtz et al. (1994), which assume spherical symmetry, and optically thin emission from a uniform density plasma with $T_{e}=10^{4} \mathrm{~K}$. The results are listed in Table 1, where column 1 is the region name, column 2 is the specific radio source, and columns 3 and 4 are the frequency $(\nu)$ and radio flux $\left(S_{\nu}\right)$, respectively. Column 5 is the observed linear size (diameter) of the radio source $(\Delta s)$ at $3 \sigma \mathrm{rms}$ level in the image, column 6 is the emission measure (EM), column 7 is the electron density $\left(n_{e}\right)$, column 8 is the excitation parameter $(U)$ and column 9 is the logarithm of the Lyman continuum flux $\left(N_{\mathrm{Ly}}^{\prime}\right)$ required for ionization. We use $\log N_{\mathrm{Ly}}^{\prime}$ to estimate the spectral type of the ionizing star (listed in column 10) using the tabulation in Panagia (1973), further assuming that a single ZAMS star is photoionizing the nebula and producing the Lyman continuum flux. The distances used for these calculations are listed in Table 4, and the near kinematic distance is adopted when the region has a distance ambiguity.

The measured sizes of the five sources listed in Table 1 are all below $0.1 \mathrm{pc}$, which according to Kurtz (2005) would suggest a classification as ultra- or hypercompact H II regions
(UC H/ HC H). However, the calculated emission measures and electron densities are an order of magnitude or more smaller than typical values for such H II regions. The typical values of the emission measure and electron density for UC H II regions are $\mathrm{EM} \gtrsim 10^{7} \mathrm{pc} \mathrm{cm}^{-6}$ and $\mathrm{n}_{e} \gtrsim 10^{4} \mathrm{~cm}^{-3}$ and for $\mathrm{HC}$ $\mathrm{H}$ II regions are $\mathrm{EM} \gtrsim 10^{10} \mathrm{pc} \mathrm{cm}^{-6}$ and $\mathrm{n}_{e} \gtrsim 10^{6} \mathrm{~cm}^{-3}$ (Kurtz 2005). We note that four of these resolved extended radio sources (18470-0044 A, 18521+0134 B, 19035+0641 B and $20293+3952 \mathrm{C}$ ) are offset $\sim 2 / 3$ of the radius from the center of the mm clumps, and are thus located at their outskirts. A plausible explanation for the lower electron densities and emission measures is that early B-type stars have formed near the edge of the dust clumps, where the density of the surrounding medium is much lower than in the center.

We will comment on two additional resolved and extended radio sources detected in this survey. The first is 18470-0044 A, which was previously observed by Hofner et al. (2011) at $25.5 \mathrm{GHz}$ using the VLA in the C-configuration. This radio source has an offset of 7 !' 2 with respect to the peak of the mm clump associated with IRAS 18470-0044 reported by Beuther et al. (2002a). Our image toward this region is affected by sidelobes due to the large flux and extended emission of a nearby radio source, and we were unable to accurately measure the radio flux at $1.3 \mathrm{~cm}$. This source is among the detections without a clear estimate of its spectral index. However, based on the flux reported by Hofner et al. (2011), and our measured flux at $6 \mathrm{~cm}$ (see Paper I), this radio source has a flat spectrum, and it is likely to be an H II region ionized by a B2 ZAMS star.

The second bright, and resolved, radio source is G34.43 $+00.24 \mathrm{~mm} 2 \mathrm{~A}$, which is the only extended source detected toward an IRDC clump in our survey. Interestingly, this radio source has a spectral index of $\alpha=-0.5$ (see Paper I) and is associated with a $24 \mu \mathrm{m}$ point source and at least two molecular outflows (Shepherd et al. 2007). This radio source was originally detected at $6 \mathrm{~cm}$ by Molinari et al. (1998, labeled by them as Mol 74) and Shepherd et al. (2004) using the VLA $\left(\right.$ FWHM $\left.\sim 6^{\prime \prime} \times 3^{\prime \prime}\right)$. The flux densities at $6 \mathrm{~cm}$ reported in those studies are consistent with our data. This radio source has an offset of $7 ! .8$ with respect to the center of the $1.3 \mathrm{~mm}$ clump $\mathrm{G} 34.43+00.24 \mathrm{~mm} 2$ detected by Rathborne et al. (2006).

We found that at least $36 \%$ of the HMCs have an extended radio source within the 1 !" 8 FWHM primary beam at $25.5 \mathrm{GHz}$. However, most of them are located slightly outside the FWHM of the mm clump and are not discussed in this study. These extended radio sources are: 18089-1732 G12.890+0.495, 18182-1433 G16.584-0.053, 18470-0044 G32.113+0.097, $18521+0134$ G34.749+0.021, $19012+0536$ G39.389-0.143 

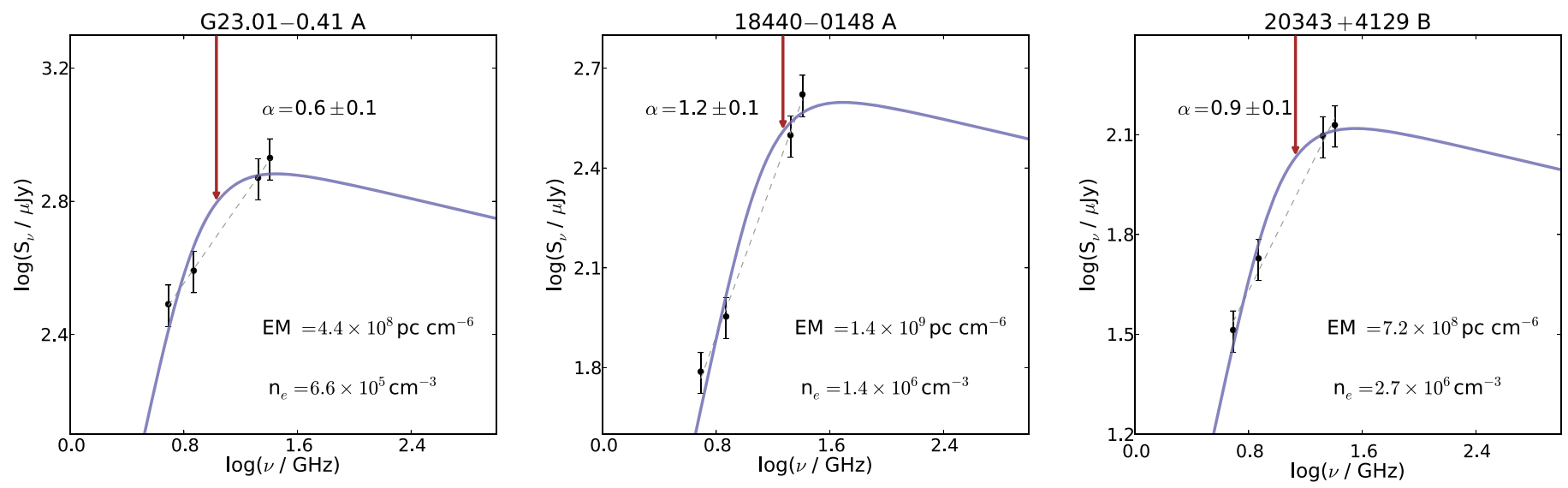

Figure 2. Spectra of the compact radio sources G23.01-0.41 A (left), 18440-0148 A (center) and 20343+4129 B (right). Error bars are an assumed uncertainty of $10 \%$ from the flux densities added in quadrature with an assumed $10 \%$ error in calibration. The continuous-blue line is the $\mathrm{H}$ II region fit using a spherical, constant density model. The red arrow indicates the frequency where $\tau_{\nu}=1$ in each model fit. The dashed line is the best fit to the data from a power-law of the form $S_{\nu} \propto \nu^{\alpha}$.

and $19266+1745$ G53.037+0.115. All of these sources and their radio continuum parameters are reported in Paper I.

\subsubsection{Compact Sources}

In Paper I we characterized the change in flux density with frequency using a power law. We reported the detection of 36 compact radio sources (51\% of total detections) with a rising spectrum $(\alpha>0.2)$, of which seven were not detected at $6 \mathrm{~cm}$, thus a lower limit for their spectral index was estimated. We also detected five compact radio sources with a flat $\mathrm{cm}$ spectrum. In this section, we investigate whether a uniform density UC/HC H II region can explain the observed fluxes and spectral indices for compact sources with rising spectra.

While optically thin free-free emission results in a flat spectral index $(\alpha=-0.1)$, a rising spectrum implies appreciable optical depth in the emitting gas. To fit our spectra, we will require a turnover (i.e., $\tau_{\nu} \sim 1$ ) near the intermediate frequency of our observing bands, around $\nu_{t}=14.7 \mathrm{GHz}$, which in turn requires an emission measure near $9 \times 10^{8} \mathrm{pc} \mathrm{cm}^{-6}$. To fit the data, we use a uniform density, spherical $\mathrm{H}$ II region model with electron temperature of $T_{e}=10^{4} \mathrm{~K}$ as shown in Equation (11) from Olnon (1975). We find that for all compact, rising spectrum sources detected in our survey, within the given uncertainties, a uniform density $\mathrm{H}$ II region spectrum can be reasonably fitted to the radio continuum data. Examples of the fits are shown by the continuous-blue line in Figure 2. The fits for all the 36 compact radio sources with rising spectral index are shown in Appendix C Figure 18.

Our fitting results show that the generally quite weak emission from these very compact, rising spectrum sources implies a very small size for the emitting regions. The sizes are much smaller than our angular resolution and are on the order of the initial Strömgren sphere radius. The initial Strömgren sphere radius $\left(R_{S}\right)$ depends on the Lyman continuum $\left(N_{\mathrm{Ly}}\right)$ flux and the ambient molecular density $\left(n_{\mathrm{H}_{2}}\right)$ as stated in Equation (1) from Xie et al. (1996):

$$
R_{s}=4104.7\left(\frac{N_{\mathrm{Ly}}}{10^{49} s^{-1}}\right)^{1 / 3}\left(\frac{n_{\mathrm{H}_{2}}}{10^{5} \mathrm{~cm}^{-3}}\right)^{-2 / 3}(\mathrm{au}) .
$$

We show the relation $R_{s}$ versus $N_{\mathrm{Ly}}$, represented by the solid lines, for $n_{\mathrm{H}_{2}}=10^{5}, 10^{6}, 10^{7}$ and $10^{8} \mathrm{~cm}^{-3}$ in Figure 3. To place our sources in this diagram, we estimated the Lyman continuum flux $\left(N_{\mathrm{Ly}}\right)$ from our $25.5 \mathrm{GHz}$ flux density, with the

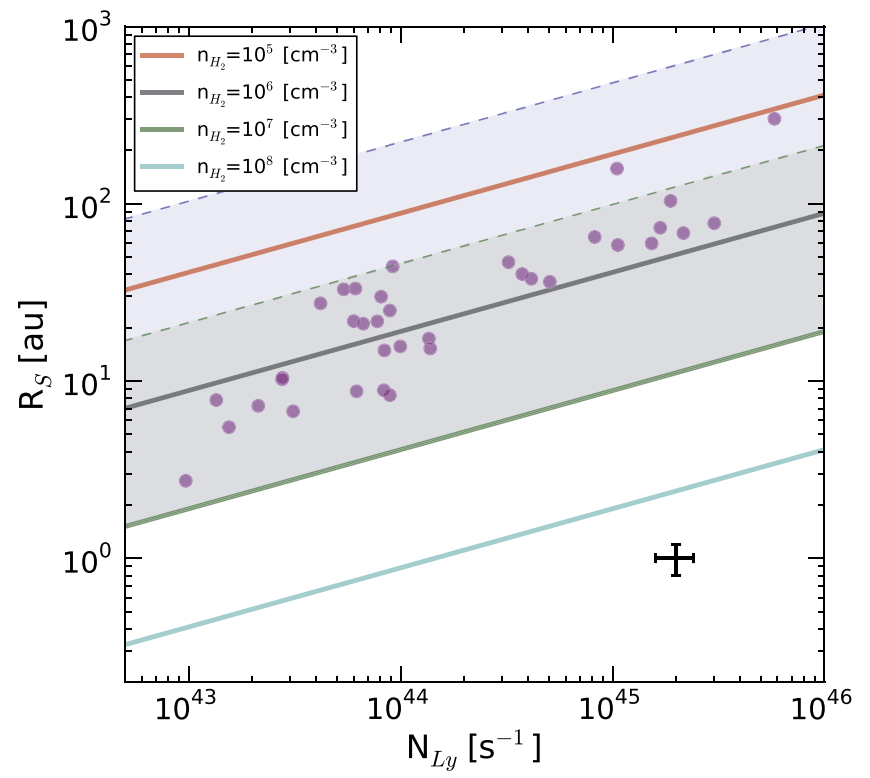

Figure 3. Initial Strömgren sphere radius as a function of the Lyman continuum for compact sources with rising spectra. The solid lines represent the ambient molecular density for $n_{\mathrm{H}_{2}}=10^{5}-10^{8} \mathrm{~cm}^{-3}$. The solid-purple dots represent the radius of the $\mathrm{H}$ II regions $(\Delta s / 2)$ as implied by the spherical, constant density $\mathrm{H}$ II region model fits. The dashed-green line represents the lower limit of $R_{\text {Turb }}$ (for $\xi=1$ ) and the dashed-blue line presents $R_{\mathrm{Th}}$ if the initial ambient molecular density for both cases is $n_{\mathrm{H}_{2}}=10^{7} \mathrm{~cm}^{-3}$. The shaded areas represent the path from their initial radius up to their final Strömgren sphere radius if the sources were born at a density of $n_{\mathrm{H}_{2}}=10^{7} \mathrm{~cm}^{-3}$ (green and blue shaded areas for turbulence and thermal pressure confinement, respectively). The error bars in the bottom right-hand corner correspond to a $20 \%$ calibration uncertainty. The data for the 36 compact sources with rising spectrum shown in this figure are available as the Data behind the figure.

(The data used to create this figure are available).

formulae of Kurtz et al. (1994), and use the radii $(\Delta s / 2)$ derived from the spectral fitting. These data are given as Data behind Figure 3. Our sources, represented as solid-purple dots in Figure 3, cluster around the expected Strömgren radius for an initial density of $10^{6} \mathrm{~cm}^{-3}$.

When nuclear burning begins and a high-mass star produces enough UV photons to photoionize the surrounding material, the initial Strömgren sphere radius is reached (to within a few percent) in a recombination timescale, $t_{r}=\left(n_{\mathrm{H}_{2}} \beta_{2}\right)^{-1}[\mathrm{~s}]$, where 
$\beta_{2}=2.6 \times 10^{-13} \mathrm{~cm}^{3} \mathrm{~s}^{-1}$ is the recombination coefficient (Dyson \& Williams 1980). For any reasonable initial density, this timescale is extremely short $(<1 \mathrm{yr})$ and the initial Strömgren sphere radius is reached almost instantaneously. The highly overpressured ionized region will then begin to expand, and hence the initial Strömgren sphere is a very shortlived configuration. Therefore, it is unlikely that the large number of sources detected represent this evolutionary stage.

After formation of the initial Strömgren sphere around a star, the UC H II region is highly overpressured and, as a result, it expands approximately at the sound speed until it approaches pressure equilibrium with the ambient medium. De Pree et al. (1995) and Xie et al. (1996) have studied the confinement of UC $\mathrm{H}$ II regions in a molecular core by thermal, and thermal plus turbulent pressure, respectively. Assuming pressure equilibrium between ionized and surrounding molecular gas, Xie et al. (1996) gives the final radius of the ionized region $(R)$ as:

$$
R=R_{s}\left(\frac{2 k \xi T_{\mathrm{H}^{+}}}{m_{\mathrm{H}_{2}} \sigma_{v}^{2}+k T_{k}}\right)^{2 / 3},
$$

where $T_{\mathrm{H}^{+}}$is the temperature of the ionized region, $\xi$ is a turbulence factor $(>1)$ that takes into account the pressure due to stellar winds and turbulence in the ionized gas, $\sigma_{v}$ is the velocity dispersion produced by turbulence and $T_{k}$ is the kinetic temperature of the surrounding molecular gas. Using typical values for the physical conditions in regions where high-mass stars form, we can test whether the sources discussed in this section could be ionized regions in pressure equilibrium with the surrounding molecular gas. While molecular line observations with single dish instruments indicate average densities of $n_{\mathrm{H}_{2}}=10^{5} \mathrm{~cm}^{-3}$ over the $1 \mathrm{pc}$ clump sizes (e.g., Hofner et al. 2000), interferometric measurements of high-mass star-forming cores have revealed central densities of $n_{\mathrm{H}_{2}}=10^{7}-10^{10} \mathrm{~cm}^{-3}$ on scales < 0.1 pc (e.g., Garay \& Rodriguez 1990; Goddi et al. 2015). Following Xie et al. (1996), we adopt values of $T_{\mathrm{H}^{+}}=$ $10^{4} \mathrm{~K}, \sigma_{v}=2 \mathrm{~km} \mathrm{~s}^{-1}\left(\mathrm{FWHM} \sim 5 \mathrm{~km} \mathrm{~s}^{-1}\right.$ ) and $T_{k}=100 \mathrm{~K}$. Assuming $\xi=1$, and evaluating the above equation with these numbers, we get $R_{\text {turb }}=11.2 R_{S}$ for the case of thermal plus turbulent pressure and $R_{\mathrm{th}}=54.3 R_{s}$ for thermal pressure only. These relations are shown in Figure 3 for $n_{\mathrm{H}_{2}}=10^{7} \mathrm{~cm}^{-3}$ as green-dashed and blue-dashed lines, respectively. Considering the location of our data points in Figure 3, we can exclude the extremely high densities of $10^{10} \mathrm{~cm}^{-3}$ as found by Goddi et al. (2015), which would predict much smaller source sizes. Meanwhile, our data points are located within the shaded areas that represent the path from their initial Strömgren radius up to their final radius in pressure equilibrium, if the sources were born at a density of $n_{\mathrm{H}_{2}}=10^{7} \mathrm{~cm}^{-3}$ (green and blue shaded areas for turbulence and thermal pressure confinement, respectively).

An estimate of the expansion time $\tau_{\text {expansion for an ionized }}$ region can be obtained assuming that it expands at its sound speed $\left(C_{s} \sim 10 \mathrm{~km} \mathrm{~s}^{-1}\right)$. To expand to $\sim 200 \mathrm{au}$, then $\tau_{\text {expansion }}$ $\sim R / C_{s} \sim 100 \mathrm{yr}$. Thus, an initial Strömgren sphere will expand fairly quickly and, as suggested by de Pree et al. (1995) and Xie et al. (1996), the ionized regions can remain compact as long as the molecular core provides the outside pressure. Observations of UC H II regions and HMCs suggest that this time is on the order of $10^{5} \mathrm{yr}$ (Wood \& Churchwell 1989a;
Wilner et al. 2001), and it hence appears that our sources could be ionized regions around newly formed stars in pressure equilibrium in their molecular cores. While our calculations have not been fitted to a particular source, the observed scatter in Figure 3 can be accounted for with a varying amount of turbulence in the molecular gas; i.e., if the molecular line FWHM varies between $\sim 7$ and $20 \mathrm{~km} \mathrm{~s}^{-1}$ for the case of an ambient molecular density of $n_{\mathrm{H}_{2}}=10^{7} \mathrm{~cm}^{-3}$.

It is interesting to note that we found that the radius of the extended sources discussed above are within the pressure equilibrium zone for an initial density of $n_{\mathrm{H}_{2}}=10^{5} \mathrm{~cm}^{-3}$. These sources are located on the outskirts of the $\mathrm{mm}$ core, and one might question whether they have migrated out of the molecular core center or if they were born in their current location. Assuming stellar velocities between 2 and $12 \mathrm{~km} \mathrm{~s}^{-1}$ (Franco et al. 2007) for them to travel to the half power point of the cores (FWHM median angular size for $\mathrm{HMC}=18^{\prime \prime}$ at a distance of $4 \mathrm{kpc}$ ), times between around $10^{5} \mathrm{yr}$ and $10^{4} \mathrm{yr}$, respectively, are needed. While migration toward lower density regions is thus possible, we note that we do not find any strong evidence for cometary regions that would be predicted due to bow shocks between molecular and ionized gas (van Buren et al. 1990).

\subsubsection{Lyman Continuum}

An additional point to consider to understand the nature of our detections is the Lyman continuum photon rate as a function of the bolometric luminosity. We analyze this relation for all the sources with a flat or a rising spectrum (including extended, elongated structure, as well as compact morphology) as shown in Figure 4. The Lyman continuum photon rate is estimated from the radio continuum flux at $25.5 \mathrm{GHz}$ and the bolometric luminosities for our regions are estimated from Herschel/Hi-GAL fluxes, and from ancillary data (see Appendix A). We list these data as Data behind Figure 4. For data taken from the literature, care was taken that the Lyman continuum flux and bolometric luminosities refer to the same distance. For sources with distance ambiguity, we use the near kinematic distance. In Figure 4, compact and elongated sources are represented by filled circles if the bolometric luminosities are estimated in this work (see Appendix A) or open circles if the luminosity is taken from the literature. The extended sources from Section 2.2.1 are represented by the $\times$ symbol. The continuous black line is the expected Lyman continuum photon rate from a ZAMS star at a given luminosity, and the shaded area bounded by the solid-black line shows the expected Lyman continuum from a stellar cluster of the same $N_{\text {Ly }}$. For more details on these curves, see SánchezMonge et al. (2013a). Thus, H II regions ionized by stellar UV photons from a single early-type star are expected to lie on the black line. Meanwhile, if the Lyman continuum comes from a cluster of stars (a likely scenario for high-mass stars) rather than from a single ZAMS star, then the expected $N_{\mathrm{Ly}}$ is lower and should be located within the shaded area (Cesaroni et al. 2015).

As seen in Figure 4, only a small fraction of our sources fall in the shaded area of the plot, which indicates direct stellar photoionization. Most of the HMC sources (red open/filled circles) lie below the curve of the expected Lyman continuum flux, and hence are underluminous at radio wavelengths. Meanwhile, the majority of the sources detected toward CMCs and CMC-IRs are located in the so-called "forbidden area" 


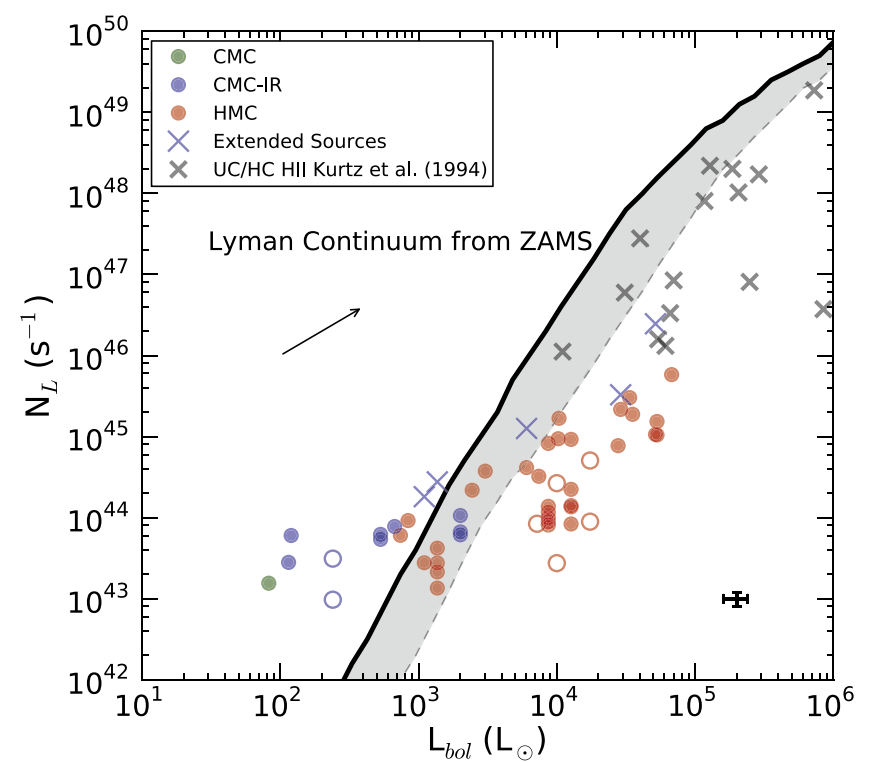

Figure 4. Lyman continuum measured at $25.5 \mathrm{GHz}$ as a function of the bolometric luminosity for all detected sources with flat or rising spectra in our sample. The bolometric luminosity is mainly estimated from $\mathrm{Herschel} / \mathrm{Hi}-$ GAL data (except for the open circles for whose the bolometric luminosity is from the literature). The circles represent the compact sources with flat or rising spectra, while the blue $\times$ symbol represents the flat spectrum extended sources from Section 2.2.1. UC H II regions from Kurtz et al. (1994) are represented by the gray $\times$ symbol. The continuous black line is the expected Lyman continuum photon rate of a single ZAMS star at a given luminosity, and the shaded area gives these quantities for the case of a cluster (Sánchez-Monge et al. 2013a). The arrow indicates how much a point would move if the distance were increased by a factor of 2 . The error bars in the bottom right -hand corner correspond to a $20 \%$ calibration uncertainty. The data for the 54 detected sources with flat or rising spectra and shown in this figure are available as the Data behind the figure.

(The data used to create this figure are available).

above the Lyman continuum line, showing an excess of Lyman continuum compared to the expected value based on their luminosities. This is true even if the sources are corrected by the distance; i.e., when there is ambiguity in the kinematic distance of the source or the value of the distance is incorrect. For reference, the arrow in the plot indicates the amount that a point will move if the distance increases by a factor of 2 . If the distance changes by any other factor, then the point will move parallel to the arrow. Additionally, there is a possibility that some bolometric luminosities are underestimated (see Appendix A). However, if this is the case, then we believe that the luminosities will shift to the right by less than 0.5 dex.

Sánchez-Monge et al. (2013a) have reported Lyman continuum excess for several sources in an 18 and $22.8 \mathrm{GHz}$ survey of high-mass star-forming regions with the ATCA. Interestingly, $\sim 70 \%$ of their $\mathrm{H}$ II regions with Lyman excess are associated with molecular clumps belonging to two types of sources that are in the earliest evolutionary stages of high-mass stars based on their classification (equivalent to our CMCs and CMC-IRs clumps). Additionally, Cesaroni et al. (2015) found Lyman continuum excess for about one-third of their sample of 200 compact and UC H II regions selected from the CORNISH survey (Purcell et al. 2013). Their sources with Lyman continuum excess are also in an earlier evolutionary phase within their sample. Both studies argued that the Lyman excess is not easily justified, which leaves room for two possible scenarios: invoking additional sources of UV photons from an ionized jet, or from an accretion shock in the protostar/disk system. Cesaroni et al. (2016) suggested that the Lyman excess is produced by accretion shocks, based on outflow $(\mathrm{SiO})$ and infall $\left(\mathrm{HCO}^{+}\right)$tracer observations toward the $200 \mathrm{H}$ II regions studied in Cesaroni et al. (2015).

It is important to mention that, due to our selection criteria (see Paper I), the sources studied by Sánchez-Monge et al. (2013a) and Cesaroni et al. (2015) are much brighter at radio wavelengths than the ones from our work, with radio luminosities at $5 \mathrm{GHz}$ of $\sim 10^{2}-10^{6} \mathrm{mJy} \mathrm{kpc}^{2}$ versus $10^{-2}-10 \mathrm{mJy} \mathrm{kpc}^{2}$ in our sample. In Figure 4, we also show several UC H II regions from Kurtz et al. (1994), denoted by the $\times$ symbol. These sources seem to be produced by higher free-free emission compared with our sample, suggesting that our sources represent a different population of radio sources. Carral et al. (1999), based on selection criteria similar to ours, detected sources with low radio luminosities like the ones in this work. Furthermore, these low radio luminosities are typical of thermal jets, with UV photons that are produced by shocks from collimated winds from the protostar with the surrounding material (e.g., Anglada 1996). Thus, while our earlier analysis of the cm spectral energy distributions (SEDs) suggests a model of pressure-confined H II regions for our compact sources, the Lyman continuum photon rate as a function of the bolometric luminosity shown in Figure 4 does not lend strong support to this model. A further possible explanation for the compact sources with rising spectra, as well as for several elongated sources detected in our survey is that they arise from thermal jets. Consequently, we will explore this scenario in the following section.

\subsection{Ionized Jets}

Based on the low radio luminosities $\left(S_{5 \mathrm{GHz}} d^{2} \sim\right.$ $10^{-2}-10 \mathrm{mJy} \mathrm{kpc}^{2}$ ) of our detected sources, we need to consider the possibility that the source of ionization is not a ZAMS star but rather that their nature is that of a thermal, ionized jet produced by shock ionization as described in Section 1. Support for this hypothesis comes from a subset of resolved sources from our survey. We have characterized 12 jet candidates based on their elongated, or string-like morphology in conjunction with an association with a molecular outflow. These sources are listed in Table 2, where column 1 is the name of the region, column 2 are the radio sources that are thought to be part of the ionized jet, and column 3 lists the approximate direction of the ionized jet. Column 4 shows the approximate direction of the molecular outflows associated with the centimeter continuum emission as found in the literature. Column 5 indicates if the centimeter continuum emission is a new detection or if it has been detected in previous studies. Column 6 lists the references for the molecular outflow detections and previous centimeter continuum detections, if any. Examples for these sources are shown in Figures 5(a), (b), and $6(a),(b)$. To our knowledge, six of these ionized jet candidates are new detections. In the cases of previous detections of centimeter continuum emission toward the listed regions, our high-sensitivity observations described in Paper I generally show the elongation or string-like morphology of a jet for the first time (e.g., Hofner et al. 2017). Furthermore, this subset of resolved jet candidates have the expected spectral index $(0.2 \leqslant \alpha \leqslant 1.2)$ for ionized jets, several of them are associated with $6.7 \mathrm{GHz} \mathrm{CH}_{3} \mathrm{OH}$ masers and $\mathrm{H}_{2} \mathrm{O}$ masers, and 
Table 2

Ionized Jets

\begin{tabular}{|c|c|c|c|c|c|c|}
\hline Region & Radio Source & Jet Direction & Outflow Direction & $\mathrm{H}_{2}-$ Jet Direction & New Detection & Reference \\
\hline $\mathrm{G} 11.11-0.12 \mathrm{P} 1$ & A, C, D & $\mathrm{NE}-\mathrm{SW}$ & $\mathrm{E}-\mathrm{W}, \mathrm{NE}-\mathrm{SW}^{\mathrm{a}}$ & $\mathrm{E}-\mathrm{W}$ & $\mathrm{y}$ & (1)-(3) \\
\hline $18089-1732$ & A & $\mathrm{N}-\mathrm{S}$ & $\mathrm{N}-\mathrm{S}$ & no/very weak ${ }^{b}$ & $n$ & (4)-(6) \\
\hline $18151-1208$ & B & $\mathrm{NE}-\mathrm{SW}$ & $\mathrm{NW}-\mathrm{SE}^{\mathrm{c}}$ & NW-SE & $\mathrm{n}$ & (7)-(10) \\
\hline IRDC18223-3 & $A-B^{e}$ & $\mathrm{NE}-\mathrm{SW}$ & $N W-S E^{f}$ & SE-NW & $\mathrm{y}$ & (14), (15) \\
\hline G23.01-0.41 & A & $\mathrm{NE}-\mathrm{SW}$ & $\mathrm{NE}-\mathrm{SW}$ & non-detection & $\mathrm{n}$ & (16)-(18), (3) \\
\hline $18440-0148$ & A & NW-SE & $\ldots \mathrm{g}$ & non-detection & $\mathrm{y}$ & (19) \\
\hline $19411+2306$ & A & $\mathrm{NE}-\mathrm{SW}$ & NE-SW & detection $^{\mathrm{b}}$ & $\mathrm{y}$ & $(24)$ \\
\hline $20126+4104$ & $A-B$ & NW-SE & $\mathrm{NW}-\mathrm{SE}, \mathrm{S}-\mathrm{N}$ & NW-SE & $\mathrm{n}$ & (25)-(29) \\
\hline $20216+4107$ & A & $\mathrm{NE}-\mathrm{SW}$ & NE-SW & $\mathrm{NE}-\mathrm{SW}$ & $\mathrm{y}$ & (23), (3) \\
\hline
\end{tabular}

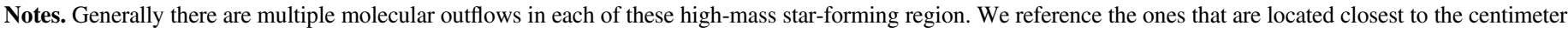
continuum emission. The " $y$ " and " $n$ " indicates if the centimeter radio continuum detection is new or if it has been detected in a previous study, respectively.

a ALMA unpublished data (V. Rosero et al., in preparation).

b T. Stanke and H. Beuther (2019, private communication).

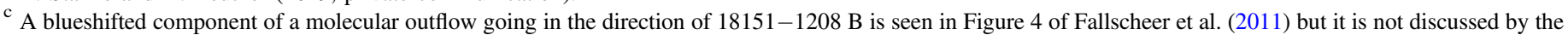
authors.

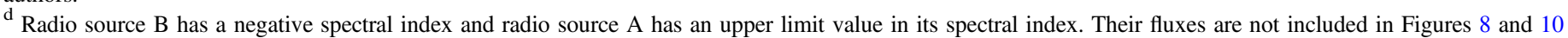
(right-hand panel).

e Radio source B has an upper limit value for the flux at $4.9 \mathrm{GHz}$ and its value is not included in Figures 8 and 10 (right-hand panel).

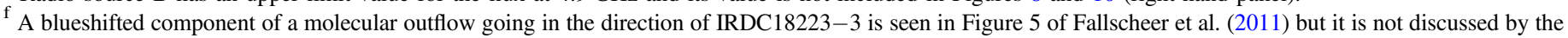
authors.

g Sridharan et al. (2002) report the presence of CO (2-1) wings toward this region, but contour maps of the molecular outflow are not available.

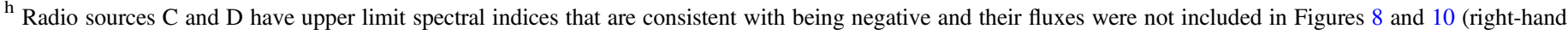
panel).

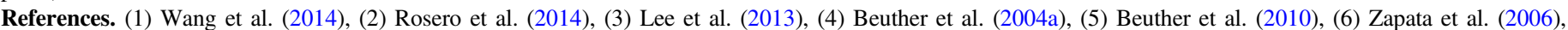

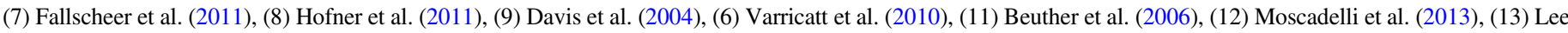

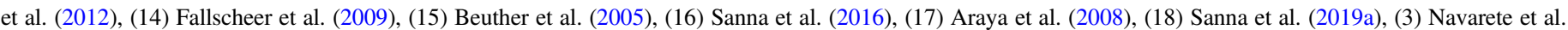

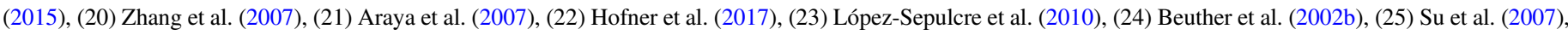
(26) Shepherd et al. (2000), (27) Hofner et al. (2007), (28) Cesaroni et al. (1999), (29) Cesaroni et al. (2013).

they have excess emission at $4.5 \mu \mathrm{m}$, which may trace shocked gas via $\mathrm{H}_{2}$ emission in outflows or scattered continuum from an outflow cavity (e.g., Cyganowski et al. 2011; Lee et al. 2013). In some cases, such as toward the ionized jet in 18182-1433, some of the radio sources have negative spectral indices, which are consistent with non-thermal lobes because it is thought that when very strong shock waves from a fast jet move through a magnetized medium, some of the electrons are accelerated to relativistic velocities producing synchrotron emission (Garay et al. 2003; Carrasco-González et al. 2010). Purser et al. (2016) and Sanna et al. $(2018,2019 b)$ also reported the detection of ionized jets with non-thermal lobes (see also the review by Anglada et al. 2018).

As listed in Table 2, at least five of the ionized jet candidates are aligned in the same direction as a large-scale molecular outflow (see Figures 5 for examples). The other ionized jet candidates appear to be associated with molecular outflows where the directions are approximately perpendicular. In Figure 6, we present the examples of 18151-1208 B and $19035+0641$ A where we show VLA $6 \mathrm{~cm}$ continuum emission contours overlaid on a $\operatorname{UKIDSS}^{13} K$-band $(2.2 \mu \mathrm{m})$ image. It is interesting to note that the putative ionized jets and the UKIDSS $K$-band emission in both cases are elongated in the same direction. This, together with the fact that the ionized jets are located nearly at the peak of the UKIDSS $K$-band

\footnotetext{
${ }^{13}$ United Kingdom Infrared Telescope (UKIRT) Infrared Deep Sky Survey (UKIDSS) Galactic Plane Survey (Lawrence et al. 2007).
}

emission, could indicate that the latter is tracing scattered light from the central protostar that is escaping from an outflow cavity (Lee et al. 2013). The observed misalignment between $\mathrm{cm}$ continuum emission and the dominating molecular flow in the region could be explained by the existence of two flows, where the molecular outflow associated with the jet is weaker, and hence undetected. This could in fact be the case for 18151-1208 B, where a blueshifted component of a CO molecular outflow observed with the Submillimeter Array appears to be aligned in the direction of the ionized jet (see Figure 4 of Fallscheer et al. 2011), although this outflow component is not discussed by the authors. Another possible explanation for the misalignment in the directions of the ionized jet and the molecular outflow is that they are subjected to precession, where the flow axis changes from the small to the large scale as suggested by, for example, Shepherd et al. (2000) and Cesaroni et al. (2005) for the case of 20126+4104, Moscadelli et al. (2013) to explain the case of 18182-1433 and Araya et al. (2007) for $18566+0408$.

For a further test of their jet nature, we have also attempted to estimate the deconvolved sizes of the central jet components using the CASA task imfit. This was possible for three sources within the subsample of jet candidates listed in Table 2 . Figure 7 shows the deconvolved major axis as a function of frequency for 18151-1208 B and 18440-0148 A (the case of $18566+0408 \mathrm{~B}$ is reported in Hofner et al. 2017). Within the uncertainties, these radio sources follow the relation $\theta_{\text {maj }} \propto \nu^{\gamma}$, where a major axis index of $\gamma=-0.7$ is expected for a 

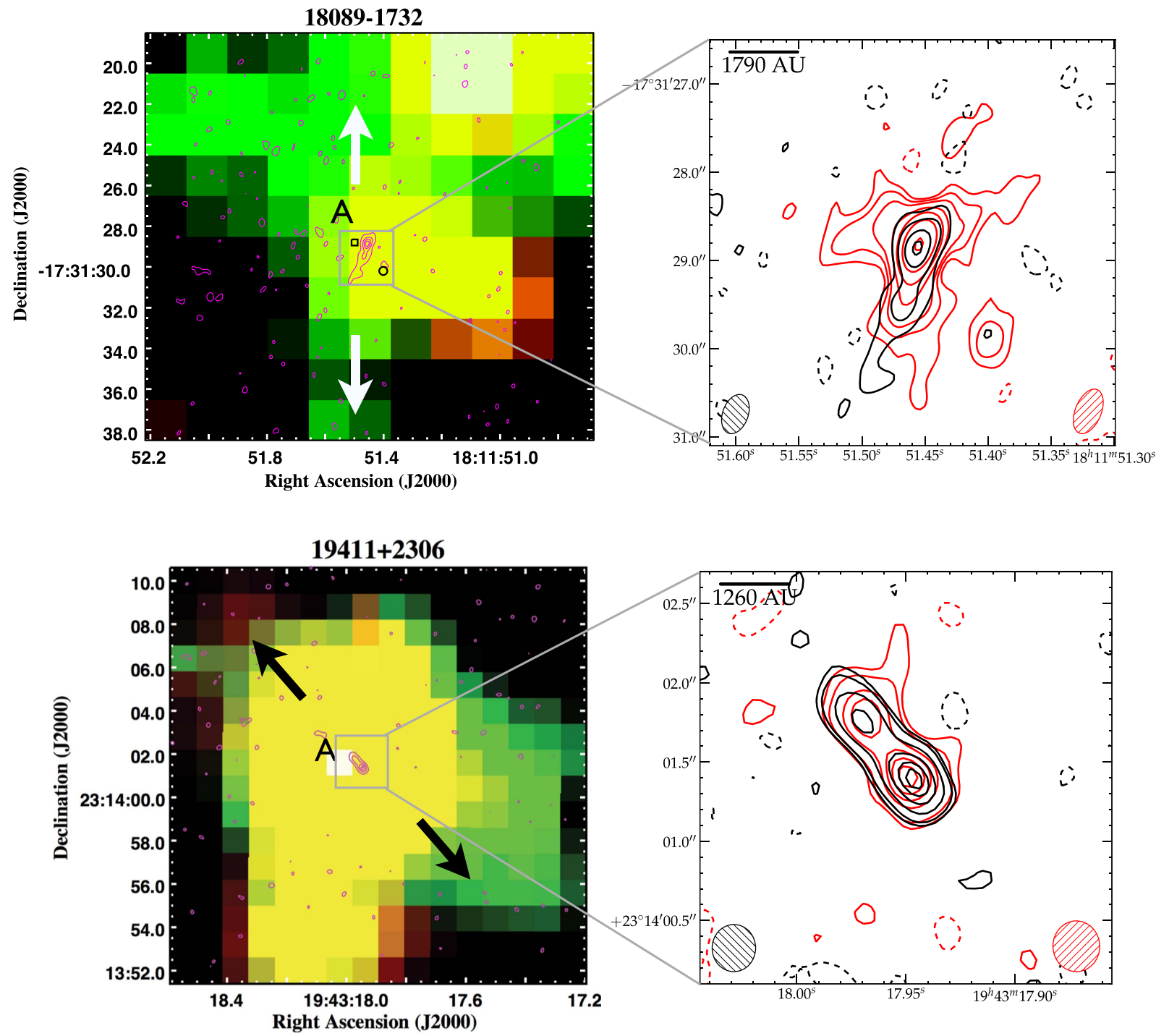

Figure 5. Spitzer IRAC GLIMPSE three-color ( $3.6 \mu \mathrm{m}$ blue, $4.5 \mu \mathrm{m}$ green and $8.0 \mu \mathrm{m}$ red) images of two ionized jet candidates, overlaid with VLA $6 \mathrm{~cm}$ continuum emission contours. Note that both regions show $4.5 \mu \mathrm{m}$ excess emission. In the right-hand panel, we show an enlarged version of the radio continuum from Paper I. Top: 18089-1732 A: the arrows represent the direction of a the north-south bipolar SiO outflow detected by Beuther et al. (2004a, 2010). The black circle and the square are the $6.7 \mathrm{GHz} \mathrm{CH} 3 \mathrm{OH}$ and $\mathrm{H}_{2} \mathrm{O}$ masers reported in (Beuther et al. 2002c), respectively. VLA $6 \mathrm{~cm}$ contour levels are $(-2.0,3.0,10.0,25.0,40.0) \times 6 \mu \mathrm{Jy}$ beam $^{-1}$, and $1.3 \mathrm{~cm}$ contour levels $(-1.5,3.0,5.0,7.5,15.0,25.0,45.0,95.0) \times 10 \mu \mathrm{Jy}$ beam $^{-1}$. Bottom: $19411+2306 \mathrm{~A}$ : the arrows represent the direction of the

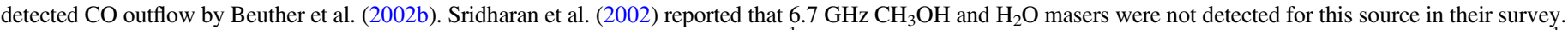
VLA $6 \mathrm{~cm}$ contour levels are $(-2.0,2.0,3.0,6.0,10.0,13.0,15.0) \times 5.5 \mu \mathrm{Jy}_{\text {beam }}{ }^{-1}$, and $1.3 \mathrm{~cm}$ contour levels $(-2.0,2.0,3.0,6.0,8.0,10.0,12.0) \times 8 \mu \mathrm{Jy}$ beam ${ }^{-1}$.

biconical ionized wind or jet (Reynolds 1986). Therefore, at least in these three cases, we have further evidence for the jet nature of these specific radio sources.

In summary, for the subsample of elongated continuum sources listed in Table 2, it is very likely that the nature of these sources are ionized jets at the base of a molecular outflow.

As mentioned earlier, most of our detected radio sources with a rising spectrum are compact; i.e., spatially unresolved, or marginally resolved. Several of these sources are associated with molecular outflows, and $6.7 \mathrm{GHz} \quad \mathrm{CH}_{3} \mathrm{OH}$ and $22 \mathrm{GHz} \mathrm{H}_{2} \mathrm{O}$ masers, as found in the literature. More precisely, only 6 of the 25 regions where we detected a radio source with a rising spectrum are not associated with molecular outflows, or adequate data that would trace such outflows do not seem to exist. Furthermore, after taking into account the shape of the synthesized beam of our VLA observations, some of these radio sources appear to be slightly elongated in a certain direction. Examples are 18264-1152 $\mathrm{F}$ and G53.25 $+00.04 \mathrm{~mm} 2$ A (see Paper I, Figure 2). Therefore, we now investigate the possibility that the compact radio continuum sources with a rising spectrum represent ionized jets.

A statistical way of investigating the nature of our compact sources is to study the energy contained in the ionized gas. In Figure 8 , we show the radio luminosity $S_{\nu} d^{2}$ of all the components of the ionized jet (or the jet candidate) as a function of the bolometric luminosity of the region. As in Figure 4, the black line is the radio luminosity expected from the Lyman continuum flux at a given bolometric luminosity if it arises from photoionization of a single ZAMS star. In addition to the compact, rising spectra sources from our survey, we also show in Figure 8 as green circles the radio luminosity from low-mass stars $\left(1 L_{\odot} \leqslant L_{\mathrm{bol}} \leqslant 1000 L_{\odot}\right)$ associated with ionized jets from Anglada et al. (2018) and as yellow circles the radio luminosity of very low luminosity objects (VeLLOs) 

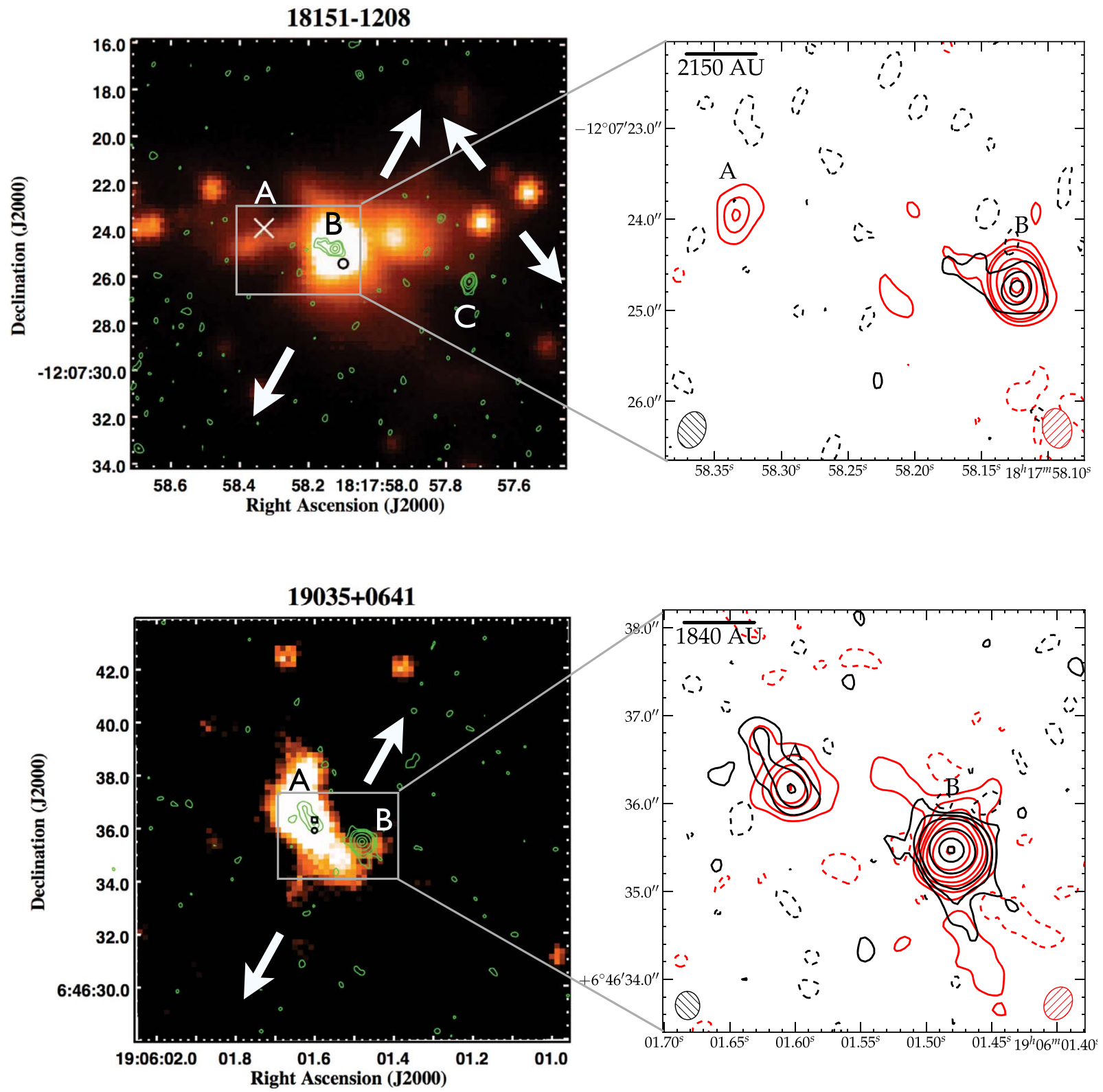

Figure 6. UKIDSS $K$-band images of two ionized jet candidates, overlaid with VLA $6 \mathrm{~cm}$ continuum emission contours. In the right-hand panel, we show an enlarged version of the radio continuum from Paper I. Top: 18151-1208 B: the arrows represent the direction of the two nearly perpendicular CO outflows detected by Fallscheer et al. (2011). A blueshifted component of a molecular outflow going in the direction of 18151-1208 B is seen in Figure 4 of Fallscheer et al. (2011) but it is not discussed by the authors. The black circle is the $6.7 \mathrm{GHz} \mathrm{CH} 3 \mathrm{OH}$ maser from Beuther et al. (2002c). The $\mathrm{x}$ symbol represents the position of an additional radio source detected at $1.3 \mathrm{~cm}$ reported in Rosero et al. (2016). VLA $6 \mathrm{~cm}$ contour levels are $(-2.0,3.0,9.0,15.0) \times 6 \mu \mathrm{Jy}$ beam ${ }^{-1}$, and $1.3 \mathrm{~cm}$ contour levels $(-2.0,3.0$, $6.0,8.5,20.0,40.0,60.0) \times 8 \mu \mathrm{Jy}_{\text {beam }}{ }^{-1}$. Bottom: $19035+0641 \mathrm{~A}$ : the arrows represent the direction of the detected $\mathrm{CO}$ and $\mathrm{HCO}^{+}$outflows $(\mathrm{Beuther}$ et al. $2002 \mathrm{~b}$; López-Sepulcre et al. 2010). The black circle and the square are the $6.7 \mathrm{GHz} \mathrm{CH}_{3} \mathrm{OH}$ and $\mathrm{H}_{2} \mathrm{O}$ masers from Beuther et al. (2002c), respectively. VLA $6 \mathrm{~cm}$ contour levels are $(-2.5,3.0,10.0,25.0,120.0,280.0,380.0) \times 4 \mu \mathrm{Jy}_{\text {beam }}^{-1}$, and $1.3 \mathrm{~cm}$ contour levels $(-2.0,5.0,10.0,20.0,30.0,50.0,90.0,170.0) \times 8 \mu \mathrm{Jy}$ beam ${ }^{-1}$.

and low-mass protostars detected at $1.8 \mathrm{~cm}$, and reported in AMI Consortium: Scaife et al. (2011, 2012). To compare the sources from AMI Consortium: Scaife et al. $(2011,2012)$ and Anglada et al. (2018) with our $4.9 \mathrm{GHz}$ data, we scaled their fluxes using a factor of 0.48 and 0.76 , respectively, assuming that those sources have a spectral index $\alpha=0.6$, which is the canonical value of ionized jets. The scaling factors are calculated using $\frac{S_{\lambda_{1}}}{S_{\lambda_{2}}}=\left(\frac{\lambda_{2}}{\lambda_{1}}\right)^{\alpha}$.

It is well-known that for low-mass YSOs, the radio luminosities are correlated with the bolometric luminosity, and we show the correlation $\frac{S_{\nu} d^{2}}{\mathrm{mJy} \mathrm{kpc}^{2}}=8.7 \times 10^{-3}\left(\frac{L_{\mathrm{bol}}}{L_{\odot}}\right)^{0.54}$ first found by Anglada (1995) and recently updated by Anglada et al. (2018). The black-dashed line is the best fit to the green circles in Figure 8, which are the low-mass ionized jets presented by Anglada et al. (2018). It is clear from Figure 8 that the sources from AMI Consortium: Scaife et al. (2011, 2012) also follow this relation, although their data were observed at low resolution $\left(\sim 30^{\prime \prime}\right)$ and there is not enough information that proves that they correspond to ionized jets. Anglada (1995) used this observed correlation to explain the apparent excess 

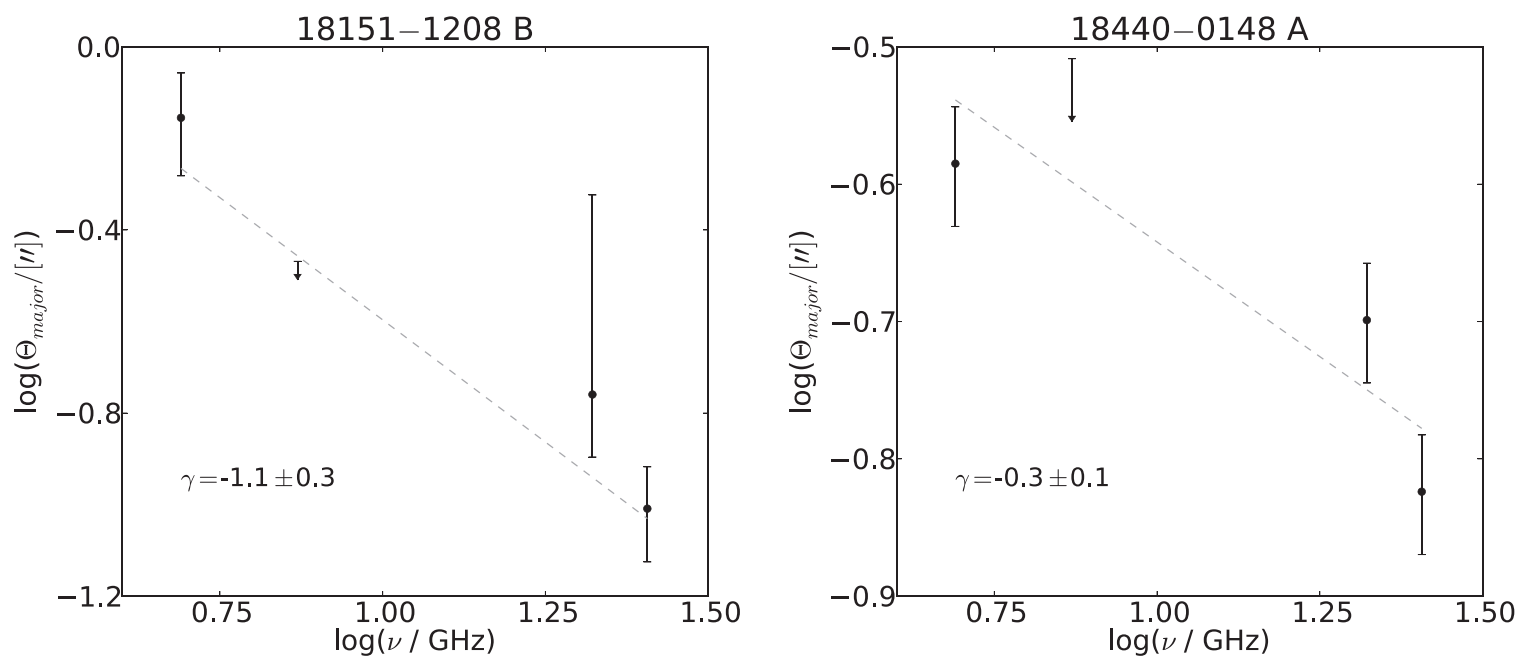

Figure 7. Deconvolved major angular axis as a function of frequency for the ionized jet candidates $18151-1208$ B, $18440-0148$ A and $18566+0408$ B. The arrows represent the size limit value from the synthesized beam of the map at the given frequency. The dashed line is the power-law fit of the form $\theta_{\text {maj }} \propto \nu^{\gamma}$.

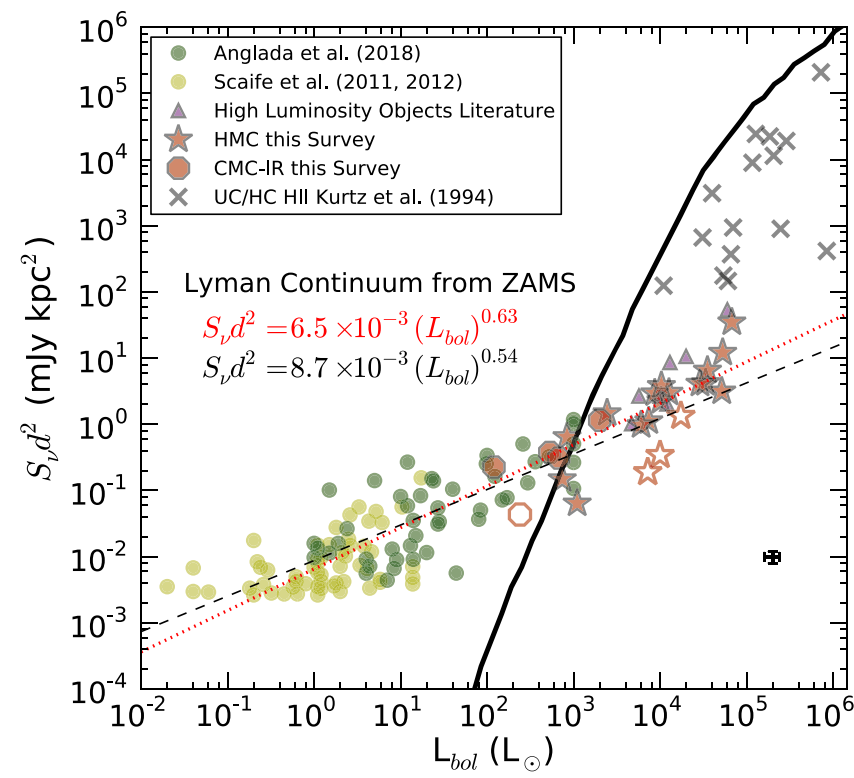

Figure 8. Radio luminosity at $4.9 \mathrm{GHz}$ as a function of the bolometric luminosity. The red stars and octagons are our ionized jet and jet candidates listed in Tables 2 and 3 toward HMCs and CMC-IRs, respectively. The bolometric luminosity for the red symbols is mainly estimated from our Herschel/Hi-GAL data (except for the open symbols whose bolometric luminosity information is from the literature). The green circles represent ionized jets associated with low-mass protostars $\left(1 L_{\odot} \leqslant L_{\mathrm{bol}} \leqslant 1000 L_{\odot}\right)$ from Anglada et al. (2018) and the yellow circles are the very low luminosity objects (VeLLOs) and low-mass protostars from AMI Consortium: Scaife et al. $(2011,2012)$. The purple triangles represent ionized jets from high-mass stars as found in the literature, from Rodríguez et al. (2008) and Moscadelli et al. (2016). The $\times$ symbols are UC and HC H II regions from Kurtz et al. (1994). The dashed line relation shows the positive correlation found by Anglada et al. (2015) derived for jets from low-mass stars. The red-dotted line is our best fit to the data including ionized jets from low, intermediate and high-mass YSOs, but excluding the sources from AMI Consortium: Scaife et al. (2011, 2012). The error bars in the bottom right corner correspond to a $20 \%$ calibration uncertainty. The data shown in this figure and in Figure 9 are available as the Data behind the figure.

(The data used to create this figure are available).

ionization levels from low-mass YSOs by shock-induced ionization from jets, as modeled by Curiel et al. (1987) and Curiel et al. (1989). A handful of detections of ionized jets toward high-mass stars in recent years have suggested that this correlation appears to also hold for stars with luminosities up to $\sim 10^{5} L_{\odot}$ (see Purser et al. 2016). We have added these objects from Rodríguez et al. (2008) and Moscadelli et al. (2016) as purple triangles in Figure 8 and the data has been properly scaled to our frequency of $4.9 \mathrm{GHz}$ assuming $\alpha=0.6$. The data from our survey (Paper I) in conjunction with improved estimates of the luminosities based on Herschel data (see Appendix A) allow us to further populate this plot and test if a correlation exists. In Figure 8, the red stars and octagons are our radio sources with rising spectrum detected toward HMCs and CMC-IRs, respectively, and we see that most sources are located very close to the relation found by Anglada (1995) up to luminosities of $\sim 10^{5} L_{\odot}$. In fact, a fit of the data including low, intermediate and high-mass YSOs is shown as a reddotted line and the result is similar to what was found by Anglada (1995). We excluded the sources from AMI Consortium: Scaife et al. $(2011,2012)$ from our fit since it is unclear if those source are indeed ionized jets. We take this result as a strong indication that the weak, and compact radio sources that we found in our survey are caused by the same mechanism which causes the radio emission the low-mass YSOs, namely it is caused by ionized jets. We also note that of the six compact radio sources where currently no observational association with molecular flows is known, five match our fit (red-dotted line in Figure 8) of the $S_{\nu} d^{2}$ versus $L_{\text {bol }}$ relationship.

Furthermore, in Figure 9 we show the momentum rate $(\dot{P})$ of the molecular outflow as a function of the radio luminosity $\left(S_{\nu} d^{2}\right)$ of the ionized jet estimated from our flux values at $4.9 \mathrm{GHz}$ (symbols and colors are the same as in Figure 8). The momentum rate of the molecular outflows comes from information from the literature for our ionized jets (and jet candidates), if available, and the values are in most cases from single dish observations. For consistency, we have scaled the physical values, so that they are based on the same distance. However, many uncertainties remain due to the inhomogeneity of the dataset. In particular, the values for the momentum rate come from observations taken by different authors, using different spectral lines, as well as different telescopes. Hence, the large scatter in Figure 9 is not unexpected, and the creation 


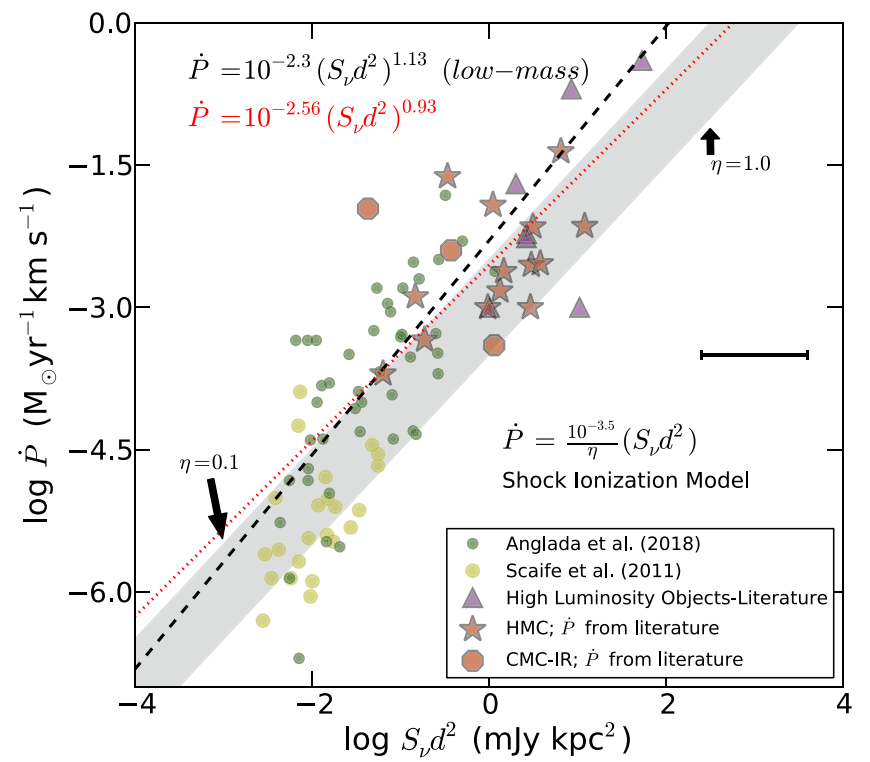

Figure 9. Momentum rate of the molecular outflow as a function of the radio luminosity at $4.9 \mathrm{GHz}$. The red stars and octagons are our ionized jets and jet candidates listed in Tables 2 and 3 toward HMCs and CMC-IRs, respectively, symbols for which there is information of the momentum rate. The momentum rate values of the molecular outflow for all the sources including our data are collected from the literature. The green circles represent ionized jets associated with low-mass protostars $\left(1 L_{\odot} \leqslant L_{\text {bol }} \leqslant 1000 L_{\odot}\right)$ from Anglada et al. (2018) and the yellow circles are the VeLLOs and low-mass protostars from AMI Consortium: Scaife et al. $(2011,2012)$. The purple triangles represent ionized jets from high-mass stars as found in the literature, from Rodríguez et al. (2008) and Moscadelli et al. (2016). The $\times$ symbols are UC and $\mathrm{HC} \mathrm{H}$ II regions from Kurtz et al. (1994). The dashed line relation shows the positive correlation found by Anglada (1995) derived for jets from low-mass stars. The red-dotted line is our best fit to the data including ionized jets from low, intermediate and high-mass YSOs, but excluding the sources from AMI Consortium: Scaife et al. $(2011,2012)$. The gray-shaded area corresponds to the momentum rate as predicted by the shock ionization model from Curiel et al. (1987) for values of the shock efficiency fraction of $\eta=0.1$ and $\eta=1.0$. The error bar in the middle right corresponds to a $20 \%$ calibration uncertainty. The error in $\dot{P}$ is not represented in the figure because it varies widely and depends strongly on how different authors have gathered the data.

of a homogenous dataset for the $\dot{P}$ versus $S_{\nu} d^{2}$ relation will be an important future task.

In spite of the large scatter, the correlation seen in Figure 9 indicates that the more radio luminous the protostars are, the more powerful they are in pushing outflowing material. This correlation, which has been studied by several authors (e.g., Anglada 1995; Rodríguez et al. 2008, AMI Consortium: Scaife et al. 2011; 2012, Anglada et al. 2018), follows the shockedinduced ionization model introduced by Curiel et al. (1987, 1989), suggesting that the ionization of thermal jets is due to shocks. The shocked-induced ionization model implies $\left(\frac{S_{\nu} d^{2}}{\mathrm{mJy} \mathrm{kpc}^{2}}\right)=10^{3.5} \eta\left(\frac{\dot{P}}{M_{\odot} \mathrm{yr}^{-1} \mathrm{~km} \mathrm{~s}^{-1}}\right)$ at $\nu=5 \mathrm{GHz}$ where $\eta$ is the shock efficiency fraction or the fraction of material that gets ionized by the shocks, which for low-mass protostars has been observationally found to be around $10 \%$ (or $\eta=0.1$ ). Anglada et al. (2018) suggested that the ionization fraction of jets in general is low $(\sim 1 \%-10 \%)$. With the current data, and due to the large scatter seen in the correlation of $\dot{P}$ versus $S_{\nu} d^{2}$, we cannot yet properly quantify how the efficiency fraction changes with the luminosity of the protostar (e.g., if the ionization in thermal jets associated with high-mass protostars is higher than for low-mass protostars). Therefore, a uniform survey to measure the momentum rate of the molecular outflows associated with ionized jets (ideally with comparable resolutions) will be fundamental to further constrain this model. Anglada et al. (2018) discussed both correlations shown in Figure 8 and Figure 9 in great detail and they interpreted them as an indication that the mechanism of ionization, accretion and ejection of outflows associated with protostars do not depend on their luminosities.

\section{Discussion}

Paper I reported the detection rates of CMC (6\%), CMC-IR (53\%) and HMCs (100\%), which provide further evidence for an evolutionary sequence in the formation of high-mass stars, from a very early stage type of cores (i.e., CMCs) to relatively more evolved ones (i.e., HMCs). The fraction of centimeter wavelength sources detected toward HMCs is higher than previously expected toward this type of cores, which suggests that radio continuum may be detectable at weak levels in all HMCs. The lack of radio detections for some objects in the sample (including most CMCs) provides interesting constraints and are ideal follow up candidates for studies of the earliest stages of high-mass stars. It is important to note that it is likely that the ionized material from jets or HC H II regions associated with these type of cores remains undetected at our sensitivity. Thus, to rule out these regions as pre-stellar cores, deeper observations are required or alternative tracers for ongoing star formation in these cores need to be identified.

Here we consider some constraints on the nature of the centimeter continuum emission detected in these cores and clumps toward high-mass star-forming regions. As described in Section 2.1, most of our radio detections arise from high-mass YSOs and at least for seven regions some of the radio detections could potentially arise from (solar-like mass) T-Tauri stars. We also detected at least 10 radio sources associated with the mm cores/clumps with a flat spectral index, most of which are resolved sources and are most likely to be UC H II regions. However, understanding the nature of the rising spectral index sources has proven to be more challenging. These compact radio sources appear to be well fitted (within the uncertainties) when using either a homogeneous $\mathrm{H}$ II region or a power-law fit (as shown in Figure 2). Therefore, we will discuss two plausible scenarios: the radio sources are either $\mathrm{UC} / \mathrm{HC} \mathrm{H}$ II regions or the emission arises from shock ionized jets.

\subsection{H II Regions}

For the first scenario, when fitting the sources in terms of a homogeneous $\mathrm{H}$ II region, the solutions required a significantly smaller size (several of them an order of magnitude smaller) for the HII region than the upper limit given by the FWHM synthesized beam. However, since these calculations assumed a pure hydrogen nebula, we must consider whether internal dust absorption can make regions that are as small as predicted by the H II region model. Wood \& Churchwell (1989b) suggested that, even if the dust absorbs $90 \%$ of the UV photons, the radius of the $\mathrm{H}$ II region is reduced by only a factor of 0.46 . Thus, dust absorption alone appears to be insufficient to explain the small region sizes predicted by the $\mathrm{H}$ II region model used to fit our data. As shown in Figure 3, these sources can be explained as turbulence-pressured confined $\mathrm{H}$ II regions if they are born in a clump with density of $n_{\mathrm{H}_{2}}=10^{7} \mathrm{~cm}^{-3}$ and assuming velocity dispersions of $\sigma \sim 3-8 \mathrm{~km} \mathrm{~s}^{-1}$ (FWHM $\sim$ 
Table 3

Ionized Jet/Wind Candidates

\begin{tabular}{|c|c|c|c|c|}
\hline Region & Radio Source & Outflow Direction & $\mathrm{H}_{2}-$ Jet Direction & Reference \\
\hline UYSO1 & A & $\mathrm{NW}-\mathrm{SE}$ & $\ldots$ & (1) \\
\hline $18264-1152$ & $\mathrm{~F}$ & $\mathrm{NW}-\mathrm{SE}$ & $E-W$ & (2), (3) \\
\hline $18345-0641$ & A & $\mathrm{NW}-\mathrm{SE}$ & very weak & (4)-(6) \\
\hline $18470-0044$ & B & E-W & no/very weak ${ }^{a}$ & (4) \\
\hline $18517+0437$ & A & $\mathrm{N}-\mathrm{S}$ & very weak & (7), (6) \\
\hline $18521+0134$ & A & $\ldots^{\mathrm{b}}$ & non-detection & (8) \\
\hline $\mathrm{G} 35.39-00.33 \mathrm{~mm} 2$ & $\mathrm{~A}$ & $\ldots$ & $\ldots$ & $\cdots$ \\
\hline $18553+0414$ & A & $\ldots{ }^{c}$ & non-detection & (3) \\
\hline $19012+0536$ & $\mathrm{~A}$ & $\mathrm{NE}-\mathrm{SW}$ & non-detection & (4), (3) \\
\hline $\mathrm{G} 53.25+00.04 \mathrm{~mm} 2$ & $\mathrm{~A}$ & $\ldots$ & $\ldots$ & $\ldots$ \\
\hline $19413+2332$ & A & $\ldots^{\mathrm{d}}$ & $\ldots$ & (4) \\
\hline $20293+3952$ & $E^{e}$ & $\mathrm{NE}-\mathrm{SW}$ & detection & $(9),(10),(6)$ \\
\hline $20343+4129$ & $\mathrm{~B}$ & E-W & non-detection & $(11),(8)$ \\
\hline
\end{tabular}

Notes. The dots indicates that there is not enough information available about observations of the molecular outflow in the literature.

T. Stanke and H. Beuther (2019, private communication).

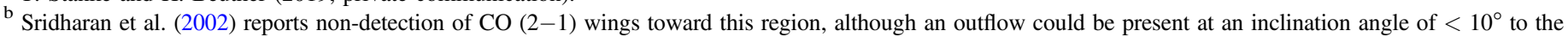
plane of the sky.

${ }^{\mathrm{c}}$ Sridharan et al. (2002) reports the presence of CO (2-1) wings toward this region, but contour maps of the molecular outflow are unavailable.

${ }^{\mathrm{d}} \mathrm{CO}$ outflow is detected in the region, but the data does not show a clear bipolar structure.

${ }^{\mathrm{e}}$ Radio source $\mathrm{E}$ has an upper limit value for the flux at $4.9 \mathrm{GHz}$ and its value is not included in Figures 8 and 10 (right-hand panel).

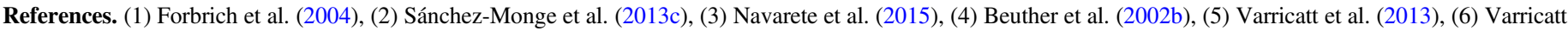
et al. (2010), (7) López-Sepulcre et al. (2010), (8) Cooper et al. (2013), (9) Beuther et al. (2004b), (10) Palau et al. (2007a), (11) Palau et al. (2007b).

$\left.7-20 \mathrm{~km} \mathrm{~s}^{-1}\right)$. However, it is not clear if high velocity dispersions are common toward the dense clumps harboring high-mass stars, since $\sigma \sim 2 \mathrm{~km} \mathrm{~s}^{-1}$ seems to be more typical. Measuring the line width of an optically thin tracer on $\sim 100 \mathrm{au}$ scale would provide a decisive constraint on the velocity dispersion. We also found that the sources could be consistent with having been born in a denser environment of $n_{\mathrm{H}_{2}}$ $\approx 10^{8} \mathrm{~cm}^{-3}$. Arguably, TTZ16 predicts that such a density for the ionized region is already too high for a protostar of $8 M_{\odot}-24 M_{\odot}$. Recently, Moscadelli et al. (2016) detected compact radio sources toward high-mass YSOs with similar physical characteristics to those found in this survey, where the Lyman continuum derived from the bolometric luminosities always exceeds the one obtained from the radio luminosities (as seen in Figure 8). From their analysis, they conclude that these sources cannot be HC or UC H II regions, unless the ionized gas has a density gradient (e.g., model IV of Olnon 1975).

Additionally, there are two scenarios for the extended UC $\mathrm{H}$ II regions detected at the outskirts of the mm cores: either they were born in a low density clump of $n_{\mathrm{H}_{2}} \approx 10^{5} \mathrm{~cm}^{-3}$ or they were born at a higher density and have migrated out of the center potential. The latter scenario requires large stellar dispersion velocities $\left(\gtrsim 10 \mathrm{~km} \mathrm{~s}^{-1}\right)$, which are not typical unless the source is a runaway $\mathrm{OB}$ star that has been dynamically ejected. For instance, the observed stellar dispersion velocities for Orion's brightest population are only $\lesssim 3 \mathrm{~km} \mathrm{~s}^{-1}$ (e.g., Sicilia-Aguilar et al. 2005; Tobin et al. 2009), which makes the former scenario more plausible. However, Franco et al. (2007) predicted that stellar velocities up to $\lesssim 13 \mathrm{~km} \mathrm{~s}^{-1}$ are likely for core densities of $10^{7} \mathrm{~cm}^{-3}$, and that these high stellar velocities carry the star to lower density regions of the core/clump, where the $\mathrm{HII}$ region is free to expand. We lean towards favoring the scenario where the sources have migrated because this can explain the occurrence of both compact and extended emission in the same protocluster (e.g., Hoare et al. 2007).

\subsection{Radio Jets}

Now we discuss the second scenario where the radio emission of the radio compact sources with rising spectrum is due to shock ionization. The observable properties of several of our radio detections indicate that they likely have a jet nature: one can argue that the low centimeter emission from the majority of the sources detected in this survey, their free-free spectral index being in the range $0.2 \leqslant \alpha \leqslant 1.8$ and their association with molecular outflows indicate that even those sources without an elongated radio morphology are also ionized jets or stellar winds that are conical, accelerating and/or recombining. From the analysis in Sections 2.2.2 and 2.3 we inferred that from the 44 sources with rising spectral index, approximately 12 of them are ionized jets (see Table 2) and 13 are jet/wind candidates (see Table 3 ). In fact, half of the jet candidates in Table 3 have a spectral index $\alpha \approx 0.6$ and all but two of them (UYSO1 A and 18521+0134 A) have a spectral index $\alpha \leqslant 1.0$, which is consistent with the expected value of a spherical, isothermal and constant velocity ionized wind (e.g., Panagia \& Felli 1975). As stated before, the deviation from the value $\alpha=0.6$ could be due to acceleration or recombination within the flow.

\subsection{H II Regions versus Radio Jets}

In Figure 8, we compared the radio luminosity with the bolometric luminosity using the radio flux at $4.9 \mathrm{GHz}$. When fitting the ionized jets (and jet candidates) from low, intermediate and high-mass protostars using a power-law (represented by the dotted red line) we find an index of $0.63 \pm 0.04$ with a correlation coefficient of $r=0.89$, which yields the relation $S_{\nu} d^{2}\left(\mathrm{mJy} \mathrm{kpc}^{-2}\right)=6.5 \times 10^{-3}$ 

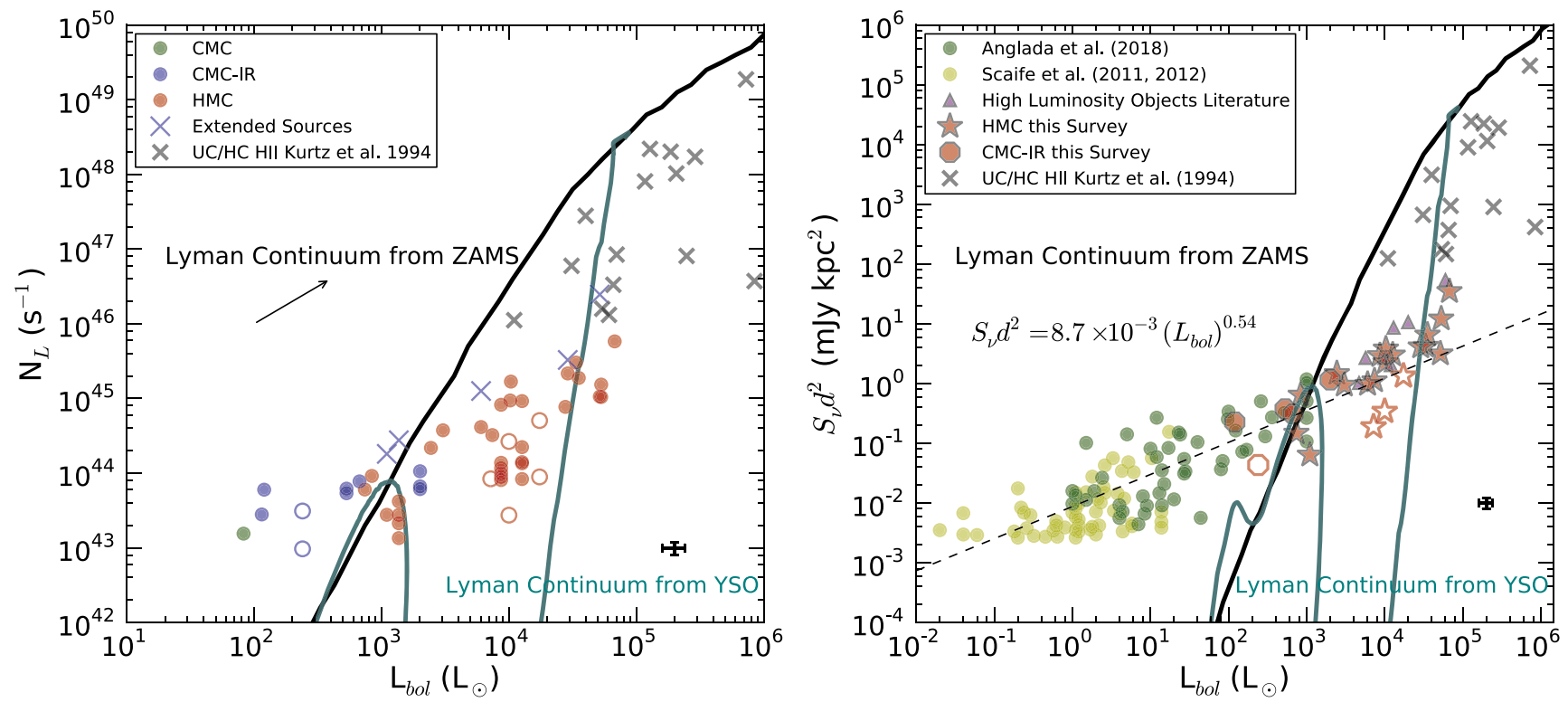

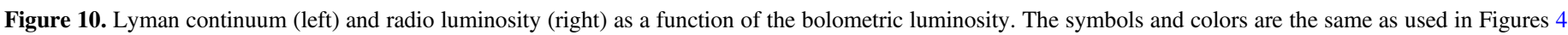

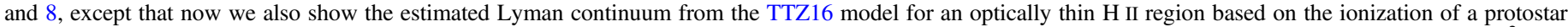

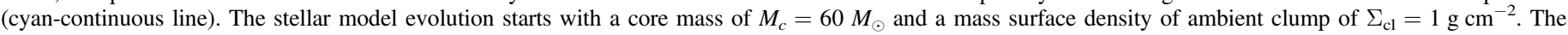
black-continuous line is the Lyman continuum from a ZAMS star. The error bars in the bottom right-hand corner correspond to a $20 \%$ calibration uncertainty.

$\left(L_{\mathrm{bol}} / L_{\odot}\right)^{0.63}$. This result is comparable with the index found by Purser et al. (2016) of $0.64 \pm 0.04$ for jets spanning luminosities from $\sim 10^{-1}$ to $10^{5} L_{\odot}$, although their fit has a lower correlation coefficient $(r=0.73)$. Their estimates for bolometric luminosities, which include Herschel/Hi-GAL data for most of their sources, are similar to ours. Therefore, the scatter in their data may come from the radio fluxes. Purser et al. (2016) have stated that some of their jets have high flux densities, probably because those objects represent a transition between a jet and H II region stages. Furthermore, it is important to note that a similar relation between the bolometric luminosity and the luminosity of shocked $\mathrm{H}_{2}$ emission from molecular jets has been reported by Caratti o Garatti et al. (2015) for sources with a wide range of bolometric luminosities. These studies together with our refined relation point to a common flow mechanism from YSOs of any luminosity.

Until very recently, the stellar evolutionary models that have been used to analyze this type of sources correspond to more evolved objects (i.e., ZAMS star). However, the recently introduced TTZ16 model predicts the ionizing luminosity of a protostar, which will allow us to compare our data with a more appropriate part of the evolutionary track. These evolutionary stellar models mainly depend on the accretion history, which is the mass of the core $\left(M_{c}\right)$ and the mass surface density of the ambient clump $\left(\Sigma_{\mathrm{cl}}\right)$. Figure 10 shows the same relations as those in Figures 4 and 8 but now we also consider an evolutionary track for a YSO, which is represented by the cyancontinuous line for an initial core mass of $M_{c}=60 M_{\odot}$ and a mass surface density of ambient clump of $\Sigma_{\mathrm{cl}}=1 \mathrm{~g} \mathrm{~cm}^{-2}$ (TTZ16 fiducial case). This cyan track shows the evolutionary sequence of the ionizing photon luminosity as a function of the protostellar luminosity. Its shape shows each of the physical stages in the evolution of the protostar: accretion stage, swelling stage (as seen with the decrease in the ionizing luminosity as the temperature decreases), contraction stage (increase of the ionizing luminosity as the temperature also increases) and nuclear burning stage when the protostar reaches the ZAMS (represented by the black-continuous line; for more discussion on this evolutionary track see Zhang et al. 2014; TTZ16). The left-hand panel of Figure 10 shows that for the majority of the radio sources detected toward CMCs and CMC-IRs, the Lyman continuum excess (for the fiducial case $L_{\text {bol }} \sim 10^{2}-10^{3}$ ) is still evident and it is unlikely to be due to photoionization. Additionally, the evolutionary track for a YSO shows how the ionizing luminosity decreases as the protostar swells while accreting its mass and before it enters the KelvinHelmholz contraction (for the fiducial case $L_{\mathrm{bol}} \sim 10^{3}-10^{4}$ ). This further indicates that the measured radio flux for most of our radio sources detected toward HMCs are also very unlikely to be photoionized by the central object.

Model calculations presented by Keto (2002, 2003, 2007) predict that high accretion rates on the order of $10^{-4}-$ $10^{-3} M_{\odot} \mathrm{yr}^{-1}$ can choke off the $\mathrm{H}$ II region to very small sizes producing very low radio continuum; see also Section 5 of Walmsley (1995). This might be a possible scenario for some of our more compact source but additional evidence is necessary, such as high-resolution $\mathrm{mm}$ observations of infall tracers to determine the mass infall rates for these sources. Based on the analysis and discussion presented here, we are inclined to favor the scenario that most of our compact sources (see Table 3) with rising spectrum are ionized jets. However, the confirmation of these radio sources as shocked ionized gas requires further observational and theoretical work. Additional observations and tests are necessary to have conclusive information of the nature of these detections. Higher resolution data $(\lesssim 0$ ". 1$)$ of the radio continuum is required to resolve the ionized jets and estimate their degree of collimation. Additionally, high resolution millimeter data will help us to disentangle multiple outflows, possibly being driven by protostellar clusters as expected toward high-mass star-forming regions and to study the kinematics of the outflow material. Masers arise from the hot core regions and their association 
with ionized material is very important. They indicate the evolutionary stage (e.g., Sanna et al. 2018) of the exciting object and they allow detailed studies of the kinematics at a smaller scale, very close to the powering high-mass YSO and the disk/jet interface. For resolved sources, long-term monitoring of the ionized jet is necessary to estimate proper motions, velocities of the radio jets and evolution in the morphology of the jet.

\section{Summary and Conclusions}

In this work we have investigated the nature of the 70 radio sources reported in Paper I. These radio sources were observed using the VLA at 6 and $1.3 \mathrm{~cm}$ toward a sample of high-mass star-forming region candidates having either no previous radio continuum detection or a relatively weak detection at the $1 \mathrm{mJy}$ level. We have explored several scenarios, such as pressureconfined H II regions and ionized jets, to explain the origin of the ionized gas emission and we have studied the physical properties of the detected sources. Based on our results, we favor the scenario that $\sim 30 \%-50 \%$ of our radio detections are ionized jets and/or jet knots. These sources, listed in Tables 2 and 3, have observational properties that are not expected toward regular $\mathrm{H}$ II regions, such as the correlation of their radio luminosity and bolometric luminosity, and the correlation of the momentum rate of the molecular outflow with the radio luminosity of the ionized jet. These correlations have been found observationally toward ionized jets associated with highmass protostars of different luminosities and they are also predicted in recent theoretical models, such as the TTZ16 model. However, for the most compact radio continuum detections, we cannot rule out the scenario that they correspond to pressure-confined $\mathrm{H}$ II regions. Our main results from this survey are summarized as follows:

1. We detected centimeter wavelength sources in $100 \%$ of our HMCs, which is a higher fraction than previously expected and suggests that radio continuum may be detectable at weak levels in all HMCs. The lack of radio detections for some objects in the sample (including most CMCs) contributes evidence that these clumps are in an earlier evolutionary stage than HMCs, providing interesting constraints and ideal follow up candidates for studies of the earliest stages of high-mass stars.

2. At least $10 \%$ of our detected radio sources are consistent with non-thermal emission and are likely to be due to either active magnetospheres in T-Tauri stars (possibly for the few regions located at a distance $<2 \mathrm{kpc}$ ) or synchrotron emission from fast shocks in disks or jets.

3 . For the most compact radio detections, the sources are consistent with being small pressure-confined H II regions. In addition, we cannot completely exclude the possibility that these sources are gravitationally trapped H II regions.

4. The majority of our detected radio continuum sources $(\sim 80 \%)$ have spectral indices $(-0.1<\alpha<2)$ that are consistent with thermal (free-free) emission from ionized gas.

5. Most of the radio sources with a rising spectrum detected toward clumps at an earlier evolutionary stage (i.e., CMCs and CMC-IRs) show Lyman continuum excess, which is consistent with previous results. This can be explained either by UV photons from shocks producing an ionized jet or shocks in an accretion flow onto the disk.

6. For most of the radio sources with a rising spectrum detected toward HMCs, the estimated Lyman continuum is lower than expected if the radio flux comes from a single ZAMS star. This may indicate that the origin of the measured radio flux is not from $\mathrm{HC} / \mathrm{UC} \mathrm{H}$ II regions but shock ionized jets.

7. We detected at least 12 ionized jets (six of them are new detections to the knowledge of the authors) based on their spectral index, morphology and molecular outflow associations. For several of the previously detected jets, we detected additional knots or lobes that are part of the collimated structure. Additionally, we detected at least 13 jet/wind candidates.

8. We found that ionized jets from low and high-mass stars are very well correlated. This is consistent with previous studies and is further evidence of a common origin for jets of any luminosity.

We thank the anonymous referee, whose comments improved this manuscript. V.R. was supported by the NRAO Grote Reber Doctoral Fellowship during this study. P.H. acknowledges support from NSF grant AST-1814011. C.C-G. acknowledges support from UNAM DGAPA-PAPIIT grant Nos. IA102816 and IN10818. L.L. acknowledges the financial support of DGAPA, UNAM (projects IN112417), and of CONACyT, Mexico. E.D.A. is partially supported by NSF grant AST-1814063. We thank K.E.I. Tanaka for providing the stellar model evolutionary tracks predicted by the TTZ16 model. We thank K.E.I. Tanaka, K. Johnston and J. Marvil for useful discussions. Herschel is an ESA space observatory with science instruments provided by European-led Principal Investigator consortia and with important participation from NASA. This work is based in part on observations made with the Spitzer Space telescope, which is operated by the Jet Propulsion Laboratory, California Institute of Technology under a contract with NASA. P.H. acknowledges support from NSF grant AST-0908901 for this project. Some of the data reported here were obtained as part of the UKIRT Service Program. The United Kingdom Infrared Telescope is operated by the Joint Astronomy Centre on behalf of the UK Particle Physics and Astronomy Research Council. This research made use of APLpy, which is an open-source plotting package for Python and is hosted at http://aplpy.github.com.

Software: CASA (McMullin et al. 2007), APLpy (Robitaille \& Bressert 2012).

\section{Appendix A Herschel/Hi-GAL Luminosity Estimates}

We obtained far-IR images from the Herschel Space Observatory (Pilbratt et al. 2010) infrared Galactic Plane Survey (Hi-GAL) project (Molinari et al. 2010) toward 52 of our regions. Hi-GAL mapped $2^{\circ}$ wide strips on the sky in the galactic longitude range $|l|<60^{\circ}$, in the five wavelength bands, $70 \mu \mathrm{m}, 160 \mu \mathrm{m}, 250 \mu \mathrm{m}, 350 \mu \mathrm{m}$ and $500 \mu \mathrm{m}$, with angular resolutions of $5^{\prime \prime}, 13^{\prime \prime}, 18^{\prime \prime}, 25^{\prime \prime}, 36^{\prime \prime}$, respectively. These data provide an improvement of a factor of $\sim 10$ in 


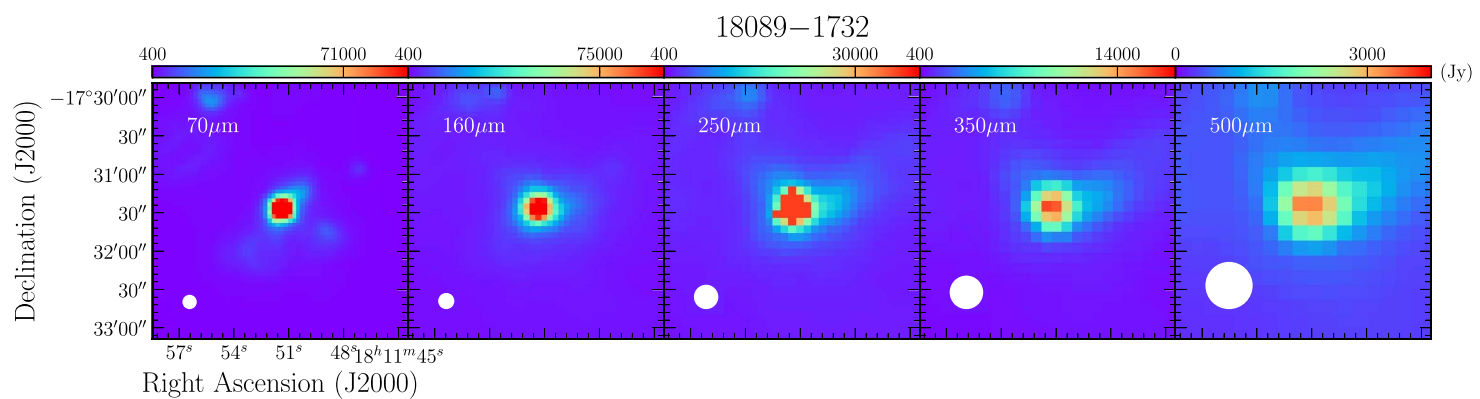

Figure 11. Hi-GAL images for 52 of the regions reported in Paper I. The complete figure set (43 images) is available in the online journal. (The complete figure set (43 images) is available.)

Table 4

SED Parameters

\begin{tabular}{|c|c|c|c|c|c|c|c|c|c|c|c|}
\hline $\begin{array}{l}\text { Region } \\
\text { (1) }\end{array}$ & $\begin{array}{c}S_{24 \mu \mathrm{m}} \\
(\mathrm{Jy}) \\
(2)\end{array}$ & $\begin{array}{c}S_{70 \mu \mathrm{m}} \\
(\mathrm{Jy}) \\
(3)\end{array}$ & $\begin{array}{c}S_{160 \mu \mathrm{m}} \\
(\mathrm{Jy}) \\
(4)\end{array}$ & $\begin{array}{c}S_{250 \mu \mathrm{m}} \\
(\mathrm{Jy}) \\
(5)\end{array}$ & $\begin{array}{c}S_{350 \mu \mathrm{m}} \\
(\mathrm{Jy}) \\
(6)\end{array}$ & $\begin{array}{c}S_{500 \mu \mathrm{m}} \\
(\mathrm{Jy}) \\
(7)\end{array}$ & $\begin{array}{c}S_{870 \mu \mathrm{m}} \\
\text { (Jy) } \\
(8)\end{array}$ & $\begin{array}{c}S_{1100 \mu \mathrm{m}} \\
(\mathrm{Jy}) \\
\text { (9) }\end{array}$ & $\begin{array}{c}\text { Distance }^{\mathrm{a}} \\
(\mathrm{kpc}) \\
(10)\end{array}$ & $\begin{array}{c}\log _{10} L^{\mathrm{b}} \\
\left(\log _{10} L_{\odot}\right) \\
(11)\end{array}$ & $\begin{array}{l}\text { Notes } \\
\text { (12) }\end{array}$ \\
\hline LDN1657A-3 & 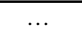 & $\ldots$ & $\begin{array}{l}\ldots \\
\cdots\end{array}$ & 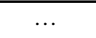 & $\ldots$ & $\ldots$ & 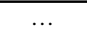 & 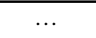 & 1.0 & $\ldots$ & $\ldots$ \\
\hline UYSO1 & $\ldots$ & $\ldots$ & $\ldots$ & $\ldots$ & $\ldots$ & $\ldots$ & $\ldots$ & $\ldots$ & 1.0 & $\ldots$ & $\ldots$ \\
\hline G11.11-0.12P1 & 1.75 & 79.88 & 133.78 & 110.59 & 68.39 & 42.79 & 15.00 & 8.56 & 3.6 & 3.31 & $\ldots$ \\
\hline $18089-1732$ & 12.31 & 1270.00 & 1580.00 & 685.30 & 452.33 & 212.59 & 45.63 & 15.25 & 2.34 & 4.01 & $\ldots$ \\
\hline $\mathrm{G} 15.05+00.07 \mathrm{~mm} 1$ & $0.10^{\mathrm{c}}$ & $2.59^{c}$ & 100.49 & 71.69 & 42.13 & 19.21 & 2.32 & 0.84 & 2.5 & 2.23 & $\ldots$ \\
\hline $18151-1208$ & $\ldots$ & $\ldots$ & $\ldots$ & $\ldots$ & $\ldots$ & $\ldots$ & $\ldots$ & $\ldots$ & 2.8 & $\ldots$ & $\ldots$ \\
\hline $\mathrm{G} 15.31-00.16 \mathrm{~mm} 3$ & 0.91 & 16.27 & $\ldots$ & 34.79 & 23.98 & 12.13 & $\ldots$ & $\ldots$ & 3.0 & 2.52 & $\ldots^{\mathrm{d}, \mathrm{e}}$ \\
\hline $18182-1433$ & 13.94 & 521.25 & 911.62 & 557.09 & 264.79 & 116.26 & 14.82 & $\ldots$ & 3.58 & 4.10 & $\ldots$ \\
\hline IRDC18223-3 & $0.31^{\mathrm{c}}$ & 6.29 & 48.75 & 82.11 & 66.91 & 32.95 & 9.48 & 2.00 & 3.7 & 2.74 & $\ldots$ \\
\hline G18.82-00.28mm3 & 0.39 & 30.19 & 107.83 & 128.05 & 70.79 & 24.91 & 10.12 & 1.60 & 3.7 & 3.11 & $\cdots$ \\
\hline $18264-1152$ & 5.60 & 373.79 & 855.74 & 614.30 & 298.49 & 111.63 & 27.37 & 8.38 & 3.3 & 3.94 & $\ldots$ \\
\hline $\mathrm{G} 22.35+00.41 \mathrm{~mm} 1$ & 0.02 & 1.26 & 37.35 & 54.43 & 41.79 & 33.20 & 7.92 & 2.46 & 3.2 & 2.43 & $\ldots$ \\
\hline $\mathrm{G} 22.73+00.11 \mathrm{~mm} 1$ & 0.82 & 15.96 & 26.71 & 31.27 & 29.34 & 17.01 & 3.33 & $\ldots$ & 4.2 & 2.82 & $(\mathrm{i})^{\mathrm{d}, \mathrm{e}}$ \\
\hline $\mathrm{G} 23.60+00.00 \mathrm{~mm} 6$ & 1.29 & 35.18 & 49.79 & 37.99 & 21.88 & 9.49 & $\ldots$ & $\ldots$ & 3.7 & 2.95 & (ii) $)^{\mathrm{d}, \mathrm{e}}$ \\
\hline G23.01-0.41 & 6.68 & 996.69 & 1560.00 & 1090.00 & 585.02 & 270.58 & 71.94 & 23.99 & 4.59 & 4.55 & $\ldots$ \\
\hline $\mathrm{G} 24.33+00.11 \mathrm{~mm} 4$ & $0.92^{\mathrm{c}}$ & $22.17^{\mathrm{c}}$ & 60.62 & 90.51 & 74.59 & 43.74 & 11.90 & 4.81 & 3.7 & 2.63 & (iii) $^{\mathrm{d}}$ \\
\hline $18337-0743$ & 19.46 & 407.39 & 342.56 & 182.35 & 87.86 & 37.94 & 4.82 & $\ldots$ & 3.8 & 3.95 & $\ldots$ \\
\hline $18345-0641$ & 9.69 & 139.83 & 199.04 & 137.33 & 67.91 & 39.19 & 4.17 & 1.13 & 5.2 & 3.87 & $\ldots$ \\
\hline $\mathrm{G} 25.04-00.20 \mathrm{~mm} 1$ & 0.53 & 21.95 & 155.96 & 253.55 & 151.45 & 92.72 & 24.55 & 6.25 & 4.3 & 3.39 & $\ldots^{\mathrm{f}}$ \\
\hline $\mathrm{G} 25.04-00.20 \mathrm{~mm} 3$ & $0.15^{\mathrm{c}}$ & $3.27^{\mathrm{c}}$ & 155.96 & 253.55 & 151.45 & 92.72 & 24.55 & 6.25 & 3.5 & 2.98 & $\ldots^{\mathrm{f}}$ \\
\hline $\mathrm{G} 27.75+00.16 \mathrm{~mm} 2$ & $0.15^{\mathrm{c}}$ & $3.75^{\mathrm{c}}$ & 16.78 & 18.89 & 19.28 & 7.28 & 1.32 & 1.02 & 3.5 & 1.94 & $\ldots$ \\
\hline $\mathrm{G} 28.23-00.19 \mathrm{~mm} 1$ & 1.14 & 25.14 & $\ldots$ & 86.22 & 71.08 & 43.17 & 15.87 & 5.11 & 4.1 & 3.09 & (i) $)^{\mathrm{d}, e, g}$ \\
\hline $\mathrm{G} 28.23-00.19 \mathrm{~mm} 3$ & $\ldots$ & $\ldots$ & $\ldots$ & 131.62 & 95.99 & 46.39 & $\ldots$ & $\ldots$ & 5.1 & 2.67 & $\ldots g$ \\
\hline $\mathrm{G} 28.53-00.25 \mathrm{~mm} 1$ & $2.10^{\mathrm{c}}$ & 43.91 & 107.09 & 245.98 & 149.55 & 79.56 & 45.41 & 26.09 & 4.4 & 3.37 & $(i v)^{\mathrm{d}, \mathrm{e}, \mathrm{h}}$ \\
\hline $\mathrm{G} 28.53-00.25 \mathrm{~mm} 2$ & $0.12^{\mathrm{c}}$ & 2.22 & 36.00 & 53.64 & 30.80 & 15.36 & $\ldots$ & $\ldots$ & 4.4 & 2.65 & $(\mathrm{v})(\mathrm{iv})^{\mathrm{h}}$ \\
\hline $\mathrm{G} 28.53-00.25 \mathrm{~mm} 4$ & $0.31^{\mathrm{c}}$ & $10.13^{\mathrm{c}}$ & 49.30 & 75.11 & 59.75 & 27.65 & 45.41 & $\ldots$ & 5.4 & 2.88 & (vi), (iv) $)^{\mathrm{h}, \mathrm{i}}$ \\
\hline $\mathrm{G} 28.53-00.25 \mathrm{~mm} 6$ & $0.31^{\mathrm{c}}$ & $10.13^{\mathrm{c}}$ & 49.30 & 75.11 & 59.75 & 27.65 & 45.41 & $\ldots$ & 5.5 & 2.90 & (vi), (iv) $)^{\mathrm{h}, \mathrm{i}}$ \\
\hline $18437-0216$ & 10.01 & 221.98 & 260.35 & 197.03 & 103.44 & 55.81 & $\ldots$ & $\ldots$ & 7.3 & 4.31 & $\ldots$ \\
\hline $18440-0148$ & 9.82 & 333.87 & 314.08 & 160.75 & 69.37 & 23.55 & 2.74 & 1.08 & 8.3 & 4.53 & $\ldots$ \\
\hline $\mathrm{G} 30.97-00.14 \mathrm{~mm} 1$ & 6.78 & 130.15 & 155.99 & 136.68 & 104.85 & 76.50 & 17.45 & 6.52 & 5.0 & 3.79 & $\ldots{ }^{\mathrm{j}}$ \\
\hline $\mathrm{G} 30.97-00.14 \mathrm{~mm} 2$ & $0.53^{\mathrm{c}}$ & $24.59^{\mathrm{c}}$ & 68.63 & 45.99 & 26.99 & 21.40 & $\ldots$ & $\ldots$ & 4.8 & 2.62 & $\left(\right.$ viii $^{\mathrm{j}}$ \\
\hline $18470-0044$ & 11.09 & 466.09 & 625.48 & 345.01 & 148.20 & 65.22 & 14.15 & 3.49 & 8.2 & 4.72 & $\ldots$ \\
\hline $\mathrm{G} 34.43+00.24 \mathrm{~mm} 1$ & 6.40 & 824.25 & 1090.00 & 545.50 & 354.29 & 200.23 & 34.74 & 34.30 & $1.56 / 3.7^{\mathrm{k}}$ & $3.49 / 4.24$ & $\ldots$ \\
\hline $\mathrm{G} 34.43+00.24 \mathrm{~mm} 2$ & 10.81 & 308.29 & 626.98 & 575.98 & 321.41 & 165.57 & 40.67 & $\ldots$ & 3.7 & 3.98 & $\ldots$ \\
\hline $18517+0437$ & $\ldots$ & $\ldots$ & $\ldots$ & $\ldots$ & $\ldots$ & $\ldots$ & 18.90 & $\ldots$ & 1.88 & $\ldots$ & $\ldots$ \\
\hline $18521+0134$ & 8.65 & 191.91 & 273.66 & 162.42 & 75.65 & 31.05 & 4.00 & 2.50 & 9.1 & 4.46 & $\ldots$ \\
\hline $\mathrm{G} 35.39-00.33 \mathrm{~mm} 2$ & 0.89 & 75.12 & 114.36 & 75.19 & 34.74 & 17.82 & 2.27 & 0.60 & 2.3 & 2.83 & $\ldots$ \\
\hline $\mathrm{G} 35.59-00.24 \mathrm{~mm} 2$ & 0.22 & 3.56 & 10.12 & 16.39 & 18.94 & 10.03 & 2.54 & 0.53 & 2.3 & 1.84 & $\ldots^{\mathrm{d}}$ \\
\hline $18553+0414$ & 13.27 & 266.24 & 289.30 & 203.20 & 69.52 & 32.75 & 5.17 & 2.02 & 12.3 & 4.83 & $\ldots$ \\
\hline $18566+0408$ & 20.27 & 722.30 & 860.88 & 561.42 & 295.14 & 156.81 & 17.65 & 5.46 & 6.7 & 4.73 & $\ldots$ \\
\hline $19012+0536$ & 6.57 & 424.28 & 402.52 & 227.30 & 93.36 & 39.49 & 5.51 & 1.97 & 4.2 & 4.02 & $\ldots$ \\
\hline $\mathrm{G} 38.95-00.47 \mathrm{~mm} 1$ & $0.28^{\mathrm{c}}$ & $2.33^{\mathrm{c}}$ & 36.69 & 62.33 & 57.17 & 38.94 & 14.71 & 5.60 & 2.1 & 2.00 & (iii) \\
\hline $19035+0641$ & 18.94 & 790.82 & 727.19 & 456.96 & 212.83 & 92.89 & 11.17 & 4.63 & 2.3 & 3.78 & $\ldots$ \\
\hline $19266+1745$ & 8.89 & 147.10 & 255.57 & 180.76 & 86.89 & 34.73 & 4.95 & $\ldots$ & 9.5 & 4.45 & $\ldots$ \\
\hline $\mathrm{G} 53.11+00.05 \mathrm{~mm} 2$ & 0.37 & 4.59 & 19.98 & 44.69 & 33.55 & 12.99 & 2.97 & $\ldots$ & 1.9 & 1.93 & $\ldots$ \\
\hline $\mathrm{G} 53.25+00.04 \mathrm{~mm} 2$ & 0.90 & 6.34 & 30.83 & 41.93 & 31.77 & 18.09 & 7.57 & 3.98 & 2.0 & 2.10 & $\ldots$ \\
\hline $\mathrm{G} 53.25+00.04 \mathrm{~mm} 4$ & 0.14 & 13.48 & 27.23 & 26.58 & 19.02 & 11.13 & $\ldots$ & $\ldots$ & 2.0 & 2.06 & $\ldots$ \\
\hline V10 & $0.11^{\mathrm{c}}$ & $1.63^{\mathrm{c}}$ & 21.49 & 25.63 & 12.49 & 6.79 & $\ldots$ & $\ldots$ & 2.3 & 1.61 & (iii) \\
\hline V11 & 0.09 & 0.53 & 4.96 & 14.89 & 12.76 & 9.86 & $\ldots$ & $\ldots$ & 2.3 & 1.51 & $\ldots$ \\
\hline V27 & $0.23^{\mathrm{c}}$ & $5.03^{\mathrm{c}}$ & 19.82 & 31.28 & 25.19 & 12.36 & $\ldots$ & $\ldots$ & 2.3 & 1.71 & (iv) \\
\hline $19411+2306$ & 15.76 & 139.80 & 188.78 & 136.78 & 68.84 & 30.06 & 8.98 & $\ldots$ & $2.9 / 5.8^{\mathrm{k}}$ & $3.39 / 3.99$ & $\ldots$ \\
\hline V33 & $0.09^{\mathrm{c}}$ & $2.41^{\mathrm{c}}$ & 18.62 & 22.13 & 14.94 & 10.76 & $\ldots$ & $\ldots$ & 2.3 & 1.59 & (iii) \\
\hline $19413+2332$ & 11.57 & 120.89 & 179.25 & 157.97 & 78.16 & 38.28 & 7.47 & $\ldots$ & $1.8 / 6.8^{\mathrm{k}}$ & $2.93 / 4.08$ & $\ldots$ \\
\hline
\end{tabular}


Table 4

(Continued)

\begin{tabular}{|c|c|c|c|c|c|c|c|c|c|c|c|}
\hline $\begin{array}{l}\text { Region } \\
\text { (1) }\end{array}$ & $\begin{array}{c}S_{24 \mu \mathrm{m}} \\
(\mathrm{Jy}) \\
(2)\end{array}$ & $\begin{array}{c}S_{70 \mu \mathrm{m}} \\
(\mathrm{Jy}) \\
(3)\end{array}$ & $\begin{array}{c}S_{160 \mu \mathrm{m}} \\
(\mathrm{Jy}) \\
(4)\end{array}$ & $\begin{array}{c}S_{250 \mu \mathrm{m}} \\
(\mathrm{Jy}) \\
(5)\end{array}$ & $\begin{array}{c}S_{350 \mu \mathrm{m}} \\
(\mathrm{Jy}) \\
(6)\end{array}$ & $\begin{array}{c}S_{500 \mu \mathrm{m}} \\
(\mathrm{Jy}) \\
(7)\end{array}$ & $\begin{array}{c}S_{870 \mu \mathrm{m}} \\
(\mathrm{Jy}) \\
(8)\end{array}$ & $\begin{array}{c}S_{1100 \mu \mathrm{m}} \\
(\mathrm{Jy}) \\
(9)\end{array}$ & $\begin{array}{c}\text { Distance }^{\mathrm{a}} \\
(\mathrm{kpc}) \\
(10)\end{array}$ & $\begin{array}{c}\log _{10} L^{\mathrm{b}} \\
\left(\log _{10} L_{\odot}\right) \\
(11)\end{array}$ & $\begin{array}{r}\text { Notes } \\
\text { (12) }\end{array}$ \\
\hline $20126+4104$ & $\cdots$ & $\cdots$ & $\cdots$ & $\cdots$ & $\cdots$ & $\cdots$ & $\cdots$ & $\cdots$ & 1.64 & $\cdots$ & $\cdots$ \\
\hline $20216+4107$ & $\cdots$ & 248.13 & 280.84 & 157.06 & 49.64 & 15.19 & $\cdots$ & $\cdots$ & 1.7 & 2.87 & $\cdots$ \\
\hline $20293+3952$ & $\cdots$ & 678.50 & 879.92 & 673.65 & 331.51 & 137.16 & $\cdots$ & $\cdots$ & $1.3 / 2.0^{\mathrm{k}}$ & $3.14 / 3.51$ & $\cdots$ \\
\hline $20343+4129$ & $\cdots$ & 468.34 & 629.64 & 452.00 & 200.76 & 77.17 & $\cdots$ & $\cdots$ & 1.4 & 3.04 & $\cdots$ \\
\hline
\end{tabular}

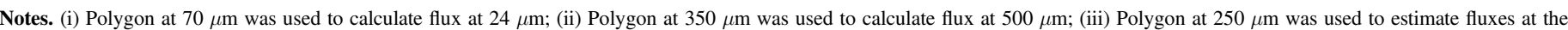

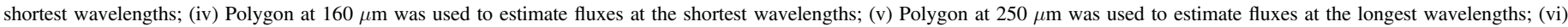

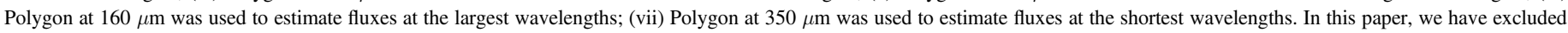
$19282+1814$ from the original survey sample. See Paper I for details.

${ }^{\text {a }}$ See Paper I for references.

b This luminosity corresponds to $L_{\text {This work }}$ plotted in Figure 15 .

${ }^{\mathrm{c}}$ Upper limit at 24 and $70 \mu \mathrm{m}$.

${ }^{\mathrm{d}}$ Absorption in $24 \mu \mathrm{m}$ map.

e Absorption in $70 \mu \mathrm{m}$ map.

${ }^{\mathrm{f}}$ Regions G25.04-00.20mm1 and G25.04-00.20mm3 are blended at wavelengths $>160 \mu \mathrm{m}$. Listed luminosities are upper limits.

${ }^{g}$ Regions G28.23-00.19mm1 and G28.23-00.19mm3 are blended.

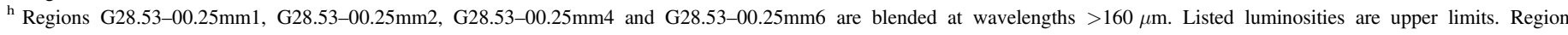
G28.53-00.25 $\mathrm{mm} 1$ is the dominant one in the map.

${ }^{\mathrm{i}}$ We used the same estimated fluxes for G28.53-00.25mm4 and G28.53-00.25mm6.

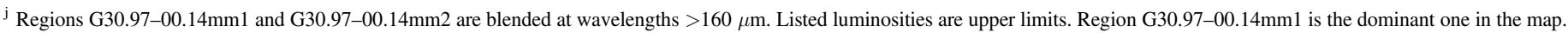
${ }^{\mathrm{k}}$ Distance ambiguity.

(This table is available in machine-readable form.)

\section{SEDs for CMCs}

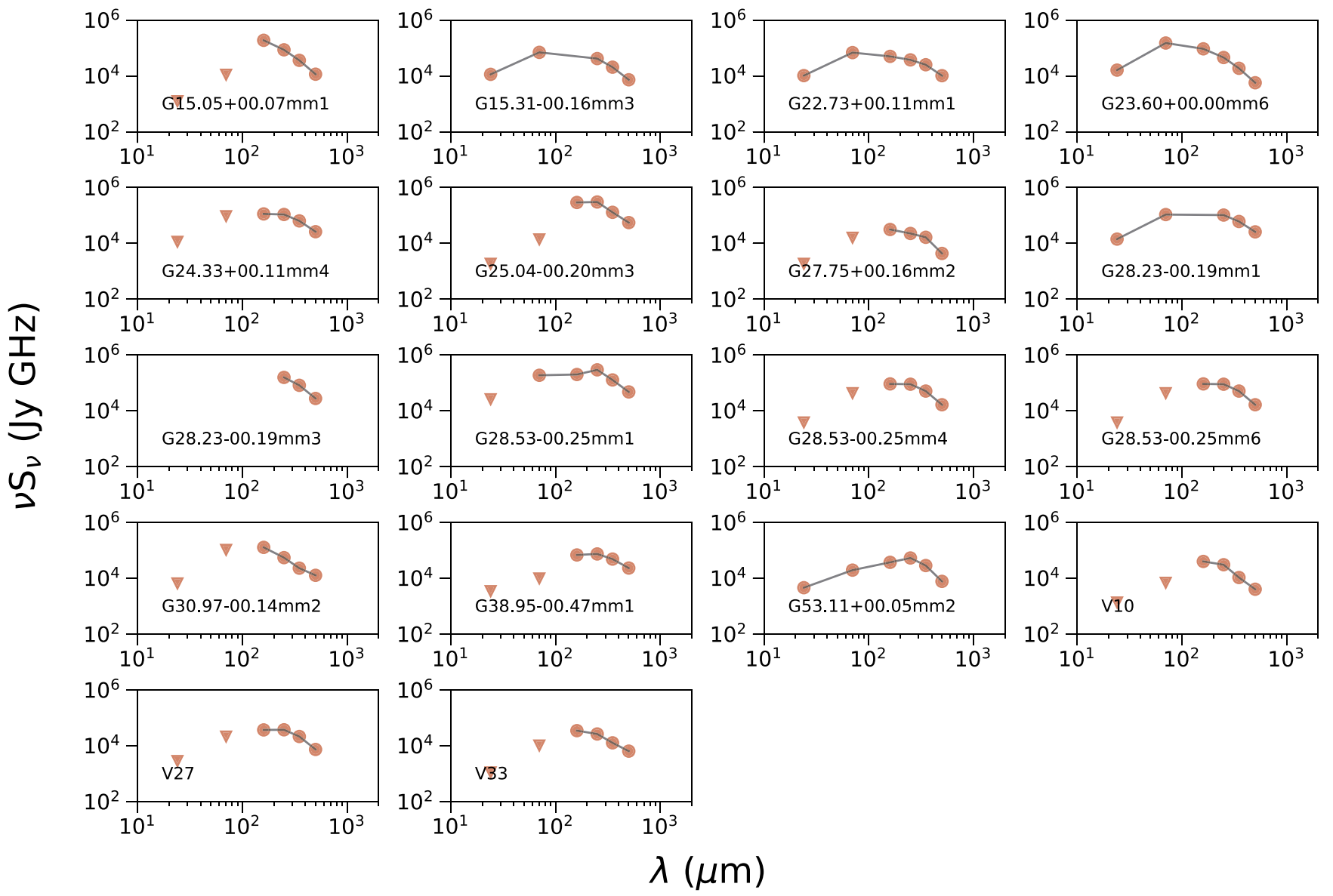

Figure 12. Spectral energy distributions of the $18 \mathrm{CMC}$ in our VLA survey with Hi-GAL counterparts. Additionally, we have included Herschel/MIPS $24 \mu$ m, ATLASGAL $870 \mu \mathrm{m}$ and BGPS v2 $1.1 \mathrm{~mm}$ flux densities. Triangles represent upper limits of the flux densities. The solid-black line represents the linear interpolation of the flux densities in the SED used to estimate the luminosity. 


\section{SEDs for CMC-IRS}
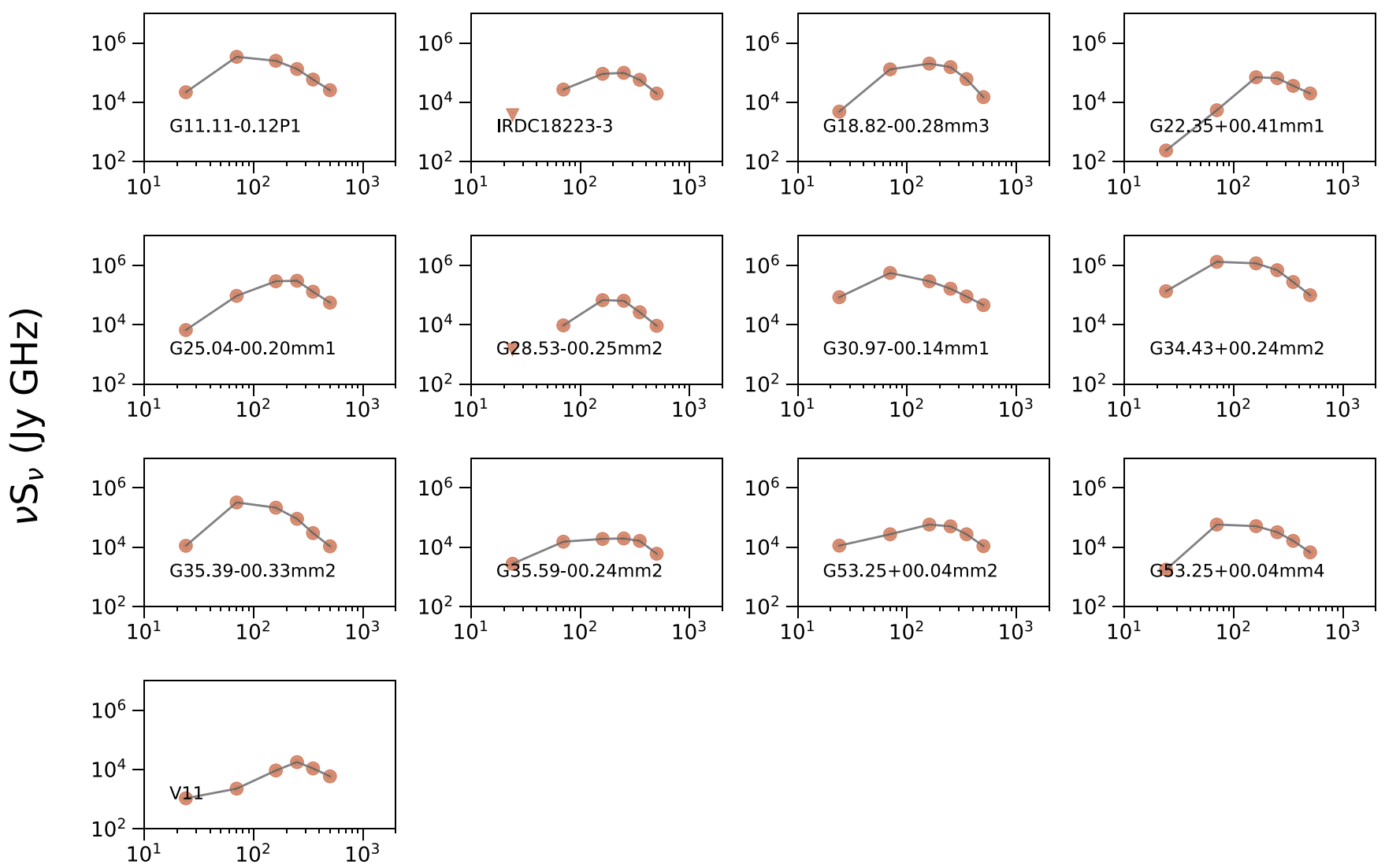

\section{$\lambda(\mu \mathrm{m})$}

Figure 13. Spectral energy distributions of $13 \mathrm{CMC}-\mathrm{IRs}$ from our VLA survey with Hi-GAL counterparts. Additionally, we have included Herschel/MIPS $24 \mu$ m, ATLASGAL $870 \mu \mathrm{m}$ and BGPS v2 $1.1 \mathrm{~mm}$ flux densities. Triangles represent upper limits of the flux densities. The solid-black line represents the linear interpolation of the flux densities in the SED used to estimate the luminosity.

spatial resolution compared to $I R A S$, thus allowing a better estimate of luminosities by lowering the contribution from unrelated nearby sources. In Figure 11, we show the HiGAL images for our targets, except for the regions LDN1657A-3, UYSO1, 18151-1208, 18517+0437 and $20126+4104$ which are located outside the region covered by $\mathrm{Hi}-\mathrm{GAL}$.

We measured the flux densities using an algorithm written in GILDAS, ${ }^{14}$ where we use a suitable polygon to enclose the source at each wavelength and integrate the flux over this area. Table 4 shows the name of the region in column 1 and the values of the flux densities are given in columns 2 through 9 . Because the angular resolution worsens with increasing wavelength, there are a number of regions where the emission appears very extended and becomes blended with nearby cores. Specifically, IRDCs G25.04-00.20, G28.23-00.19, G28.53 -00.25 and G30.97-00.14 have millimeter cores that are highly blended; for these sources, we only report an upper limit

\footnotetext{
$\overline{14}$ http://www.iram.fr/IRAMFR/GILDAS
}

for the luminosities. In addition, for some regions we can only estimate lower or upper limits for the flux densities at 24 and $70 \mu \mathrm{m}$, due to absorption or non-detection. In the latter case, we do not include these data points when estimating the luminosity. The specific cases are identified in Table 4 column 12 or are pointed out with footnotes. Additionally, for a handful of sources, there was not enough ancillary data available and therefore their bolometric luminosity might be underestimated (e.g., 20216+4107, 20293+3952 and 20343+4129).

We also gathered images at $24 \mu \mathrm{m}$ from Spitzer/MIPS (Rieke et al. 2004) for the construction of the SEDs for our targets. The Spitzer/MIPS data are from the Multiband Imaging Photometer for Spitzer Galactic Plane Survey (MIPSGAL; Carey et al. 2005). Sub-mm fluxes obtained from the APEX Telescope Large Survey of the Galaxy (ATLAS$\mathrm{GAL}^{15}$ ) (Urquhart et al. 2014) and Bolocam Galactic Plane Survey (BGPS) v2 (Ellsworth-Bowers et al. 2015) taken at

\footnotetext{
15 The ATLASGAL project is a collaboration between the Max-PlanckGesellschaft, the European Southern Observatory (ESO), and the Universidad de Chile.
} 


\section{SEDs for HMCs}
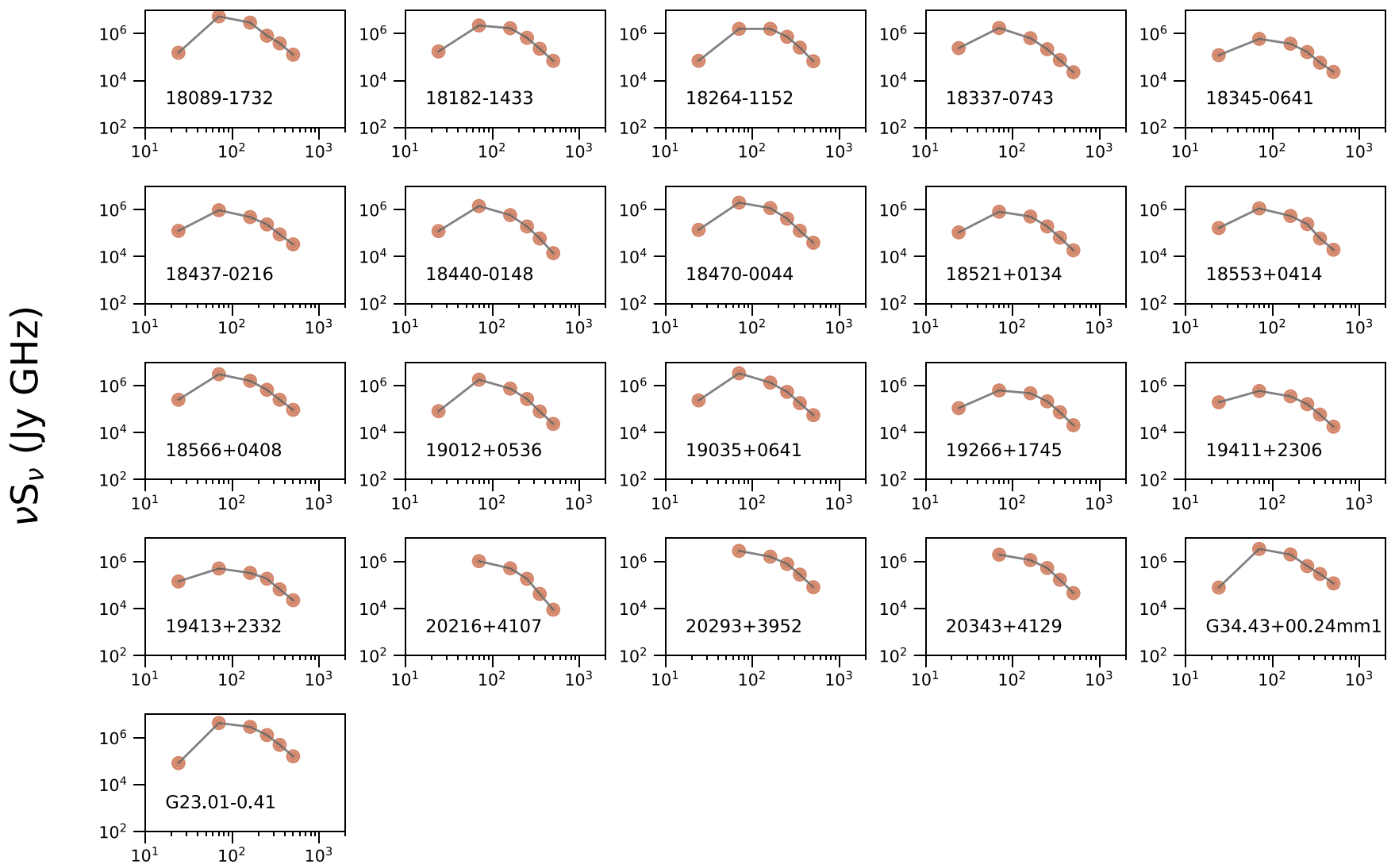

\section{$\lambda(\mu \mathrm{m})$}

Figure 14. Spectral energy distributions of 23 HMC from our VLA survey with Hi-GAL counterparts. Additionally, we have included Herschel/MIPS $24 \mu$ m, ATLASGAL $870 \mu \mathrm{m}$ and BGPS v2 $1.1 \mathrm{~mm}$ flux densities. Triangles represent upper limits of the flux densities. The solid-black line represents the linear interpolation of the flux densities in the SED used to estimate the luminosity.

$870 \mu \mathrm{m}$ and $1.1 \mathrm{~mm}$, respectively, are also used to estimate the luminosities. Figures 12-14 show the SEDs of the CMCs, CMC-IRs and HMCs of 52 out of the 57 regions observed with the VLA. We estimated the luminosity of each region integrating the continuum spectra by linearly interpolating the flux densities in the SED. The distance used to calculate the luminosities and the estimated luminosities are given in Table 4 columns 10 and 11, respectively.

In Figure 15, we compare the luminosities derived in this work with values from the literature. For the case of HMCs (red points) the literature values are mostly based on far-infrared data taken with the Infrared Astronomical Satellite (IRAS) database (Sridharan et al. 2002). In almost all cases, our luminosities are smaller than the IRAS luminosities. This is likely to be due to the inclusion of extended emission or unrelated sources in the large IRAS beams. However, the difference is usually $<0.3$ dex.
For the CMCs and CMC-IRs (green and blue points), the literature values for the luminosities were mostly taken from Rathborne et al. (2010), who constructed SEDs using a combination of Spitzer and ground based telescope data. For these sources, the scatter in Figure 15 is somewhat larger than for HMCs. This could be due to a variety of factors; however, most sources have a consistent luminosity within a factor of $<0.5$ dex.

For the analysis performed in this work, we have used the luminosities that we have derived as described earlier, with exception of sources where we only derived limits (G25.04-00.20mm1, G25.04-00.20mm3, G28.23-00.19mm1, G28.23-00.19mm3, G28.53-00.25mm1, G28.53-00.25mm2, G28.53-00.25mm4, G28.53-00.25mm6, G30.97-00.14mm1 and G30.97-00.14mm2) or those sources that do not have HI-GAL data (see above). In both cases, if available, we used the literature values instead. 


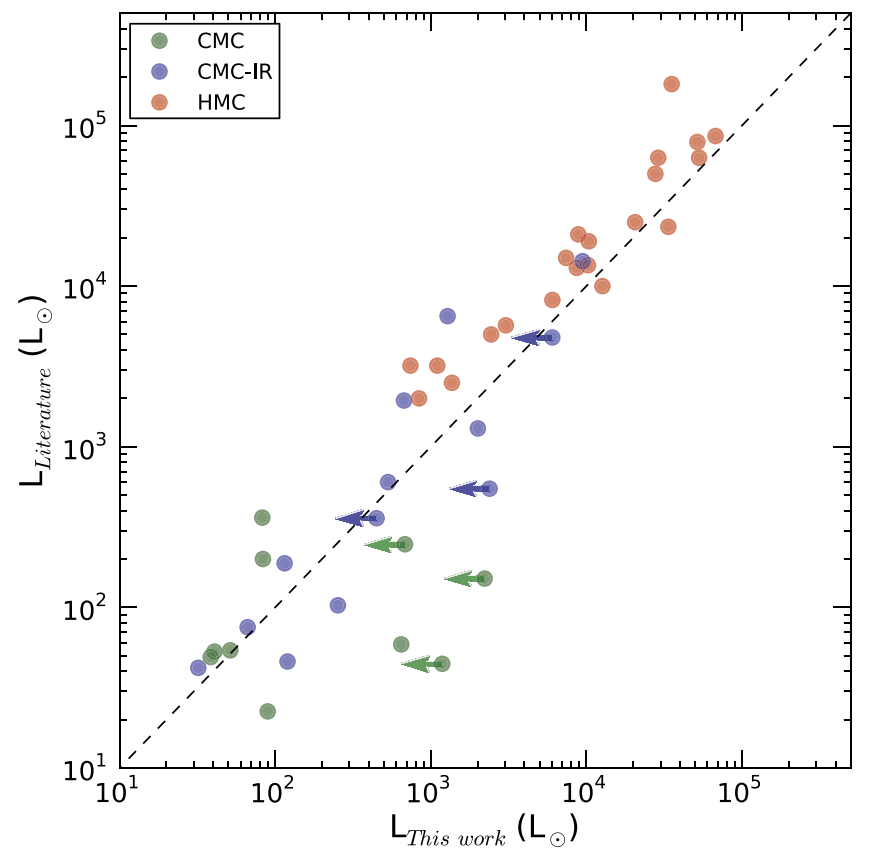

Figure 15. Comparison between our luminosity estimates with respect to luminosities from the literature. Most of the previous luminosities estimated for the HMCs in our sample are IRAS luminosities. The dashed line corresponds to $L_{\text {Lit }}=L_{\text {This work. }}$. The arrows represent upper limits of our luminosity calculations for the given region. We use the near distance for sources with distance ambiguity. The values for $L_{\text {This work }}$ and $L_{\text {Lit }}$ are presented in Table 4 and Paper I Table 1, respectively.

\section{Appendix B}

\section{Understanding the Momentum Rate of the Ionized Jet}

If we assume that our radio sources with rising spectrum have an ionized jet nature, then we can use the models of Curiel et al. (1987) and Reynolds (1986) to derive some interesting parameters of the jet to understand its behavior. From the shock- induced ionization model assuming a plane-parallel shock, Curiel et al. (1987) and Curiel et al. (1989) derived a relationship between the momentum rate of the molecular outflow $\left(\dot{P}_{\text {Curiel }+}\right)$ and the centimeter emission from the ionized jet given by Curiel et al. (1989), Anglada et al. (1998), Johnston et al. (2013):

$$
\begin{aligned}
& \left(\frac{\dot{P}_{\text {Curiel }+}}{M_{\odot} \mathrm{yr}^{-1} \mathrm{~km} \mathrm{~s}^{-1}}\right)=\frac{3.13 \times 10^{-4}}{\eta}\left(\frac{S_{\nu} d^{2}}{\mathrm{mJy} \mathrm{kpc}}\right) \\
& \quad \times\left(\frac{v_{\star}}{200 \mathrm{~km} \mathrm{~s}^{-1}}\right)^{0.32}\left(\frac{T}{10^{4} \mathrm{~K}}\right)^{-0.45} \\
& \quad \times\left(\frac{\nu}{5 \mathrm{GHz}}\right)^{0.1}\left(\frac{\tau}{1-e^{-\tau}}\right),
\end{aligned}
$$

where $\eta$ is the shock efficiency fraction ${ }^{16}$ observationally found to be only partial with $\eta \sim 0.1$ for low-mass stars (e.g., Anglada 1995; Anglada et al. 1998). The initial velocity of the jet is $v_{\star}$ which for high-mass stars is $\sim 700 \mathrm{~km} \mathrm{~s}^{-1}$ (e.g., Rodriguez et al. 1994), $T$ is the temperature of the ionized gas which is assumed to be $10^{4} \mathrm{~K}, S_{\nu}$ is the observed flux density at a frequency $\nu$ and $\tau$ is the optical depth of the ionized gas. If

\footnotetext{
16 The shock efficiency $\eta=\frac{\Omega}{4 \pi}$ is the fraction of the solid angle that is shocked (Anglada 1996).
}

the flux is measured also at another frequency, $\nu^{\prime}$, one can measure the spectral index $\alpha$ and obtain $\tau$ from the expression:

$$
\begin{aligned}
\alpha & =2+\frac{1}{\ln \left(\frac{\nu}{\nu^{\prime}}\right)} \ln \\
& \times\left[\frac{1-\frac{2}{\tau^{2}}\left\{1-(\tau+1) e^{-\tau}\right\}}{1-\frac{2}{\left[\tau\left(\frac{\nu}{\nu^{\prime}}\right)^{2.1}\right]^{2}}\left\{1-\left(\tau\left(\frac{\nu}{\nu^{\prime}}\right)^{2.1}+1\right) e^{\left.-\tau\left(\frac{\nu}{\nu^{\prime}}\right)^{2.1}\right\}}\right.}\right] .
\end{aligned}
$$

This relationship is equivalent to the one derived by Anglada et al. (1998) for a cylindrical distribution.

Similarly, the momentum rate $\left(\dot{P}_{\text {Reynolds }}\right)$ from a partially optically thick jet can be estimated using the model from Reynolds (1986):

$$
\begin{aligned}
& \left(\frac{\dot{P}_{\text {Reynolds }}}{M_{\odot} \mathrm{yr}^{-1} \mathrm{~km} \mathrm{~s}^{-1}}\right)=9.38 \times 10^{-6}\left(\frac{v_{\text {jet }}}{100 \mathrm{~km} \mathrm{~s}^{-1}}\right)^{2}\left(\frac{1}{x_{0}}\right) \\
& \quad \times\left(\frac{\mu}{m_{p}}\right)\left[\left(\frac{S_{\nu}}{\mathrm{mJy}}\right)\left(\frac{\nu}{10 \mathrm{GHz}}\right)^{-\alpha}\right]^{0.75} \\
& \quad \times\left(\frac{d}{\mathrm{kpc}}\right)^{1.5}\left(\frac{\nu_{m}}{10 \mathrm{GHz}}\right)^{-0.45+0.75 \alpha} \\
& \quad \times \theta_{0}^{0.75}\left(\frac{T}{10^{4} \mathrm{~K}}\right)^{-0.075}(\sin i)^{-0.25} F^{-0.75} .
\end{aligned}
$$

Most of these parameters are not known, except for $\alpha$ and $S_{\nu}$ measured at $\nu=4.9 \mathrm{GH}$. Thus, for the rest of parameters, we assume values that are typical of jets associated with luminous objects. The expanding velocity of the jet $v_{\text {jet }}=700 \mathrm{~km} \mathrm{~s}^{-1}$, the opening angle $\theta_{0}=1 \mathrm{rad}$, the ionized gas temperature $T=10^{4} \mathrm{~K}$, the inclination with the line of sight $i=45^{\circ}$, the hydrogen ionization fraction $x_{0}=0.1$ and the ratio of the mean particle mass per hydrogen atom and the proton mass $\mu / m_{p}=1 /\left(1+x_{0}\right)$ (Rodriguez et al. 1994). The turnover frequency value that we adopted for our calculations is $\nu_{m}=50 \mathrm{GHz}$, which is the highest frequency of the VLA Q-band receiver. The parameter $F$ which is an index for the jet optical depth is estimated from Equation (17) of Reynolds (1986):

$$
F=\frac{(2.1)^{2}}{q_{\tau}(\alpha-2)(\alpha+0.1)},
$$

and assuming the parameters for the standard case (isothermal, constant velocity and fully ionized jet) with $q_{\tau}=-3 \epsilon$ and $\epsilon=\frac{0.7}{1.3-\alpha}$ (see Section 1).

Figure 16 shows the momentum rate of the molecular outflow $\left(\dot{P}_{\text {outflow }}\right)$ versus the momentum rate of an associated partially optically thick ionized jet ( $\left.\dot{P}_{\text {Reynolds }}\right)$ as modeled by Reynolds (1986) at $\nu=4.9 \mathrm{GHz}$ assuming $\alpha=0.6$ and the same parameters listed earlier. The green and gray circles represent ionized jets associated with low-mass $\left(1 L_{\odot} \leqslant\right.$ $\left.L_{\text {bol }} \leqslant 1000 L_{\odot}\right)$ and high-mass $\left(L_{\text {bol }}>1000 L_{\odot}\right)$ protostars from Anglada et al. (2018), respectively, scaled to a frequency of $\nu=4.9 \mathrm{GHz}$. The momentum rate of the molecular outflows is taken from the literature, usually from single dish observations. The red and blue circles are our radio detections 


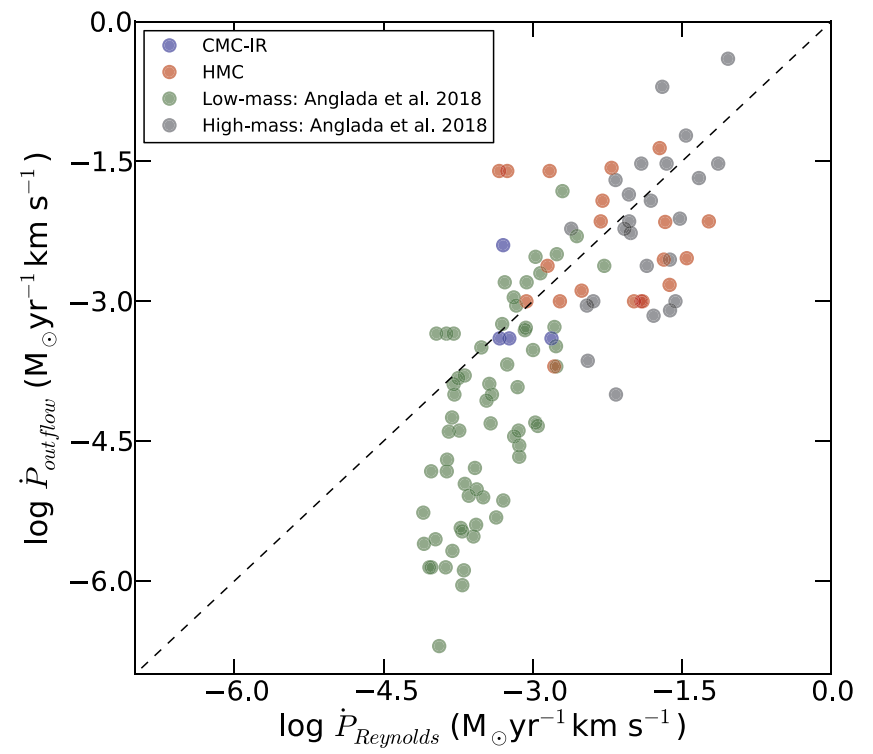

Figure 16. Momentum rate of the molecular outflow vs. the momentum rate of an associated partially optically thick ionized jet as modeled by Reynolds (1986) at $\nu=4.9 \mathrm{GHz}$. The green and gray circles represent ionized jets associated with low-mass $\left(1 L_{\odot} \leqslant L_{\text {bol }} \leqslant 1000 L_{\odot}\right)$ and high-mass $\left(L_{\text {bol }}>1000 L_{\odot}\right)$ protostars from Anglada et al. (2018), respectively. The momentum rate of the molecular outflows is data from the literature, usually from single dish observations. The dashed line represents the case where the momentum rate of the molecular outflow and the momentum rate of the ionized jet are the same. The momentum rate of the molecular outflow and the momentum rate of the associated ionized jet as estimated using the model of Reynolds (1986) is significantly similar for sources with $L_{\text {bol }} \gtrsim 100 L_{\odot}$.

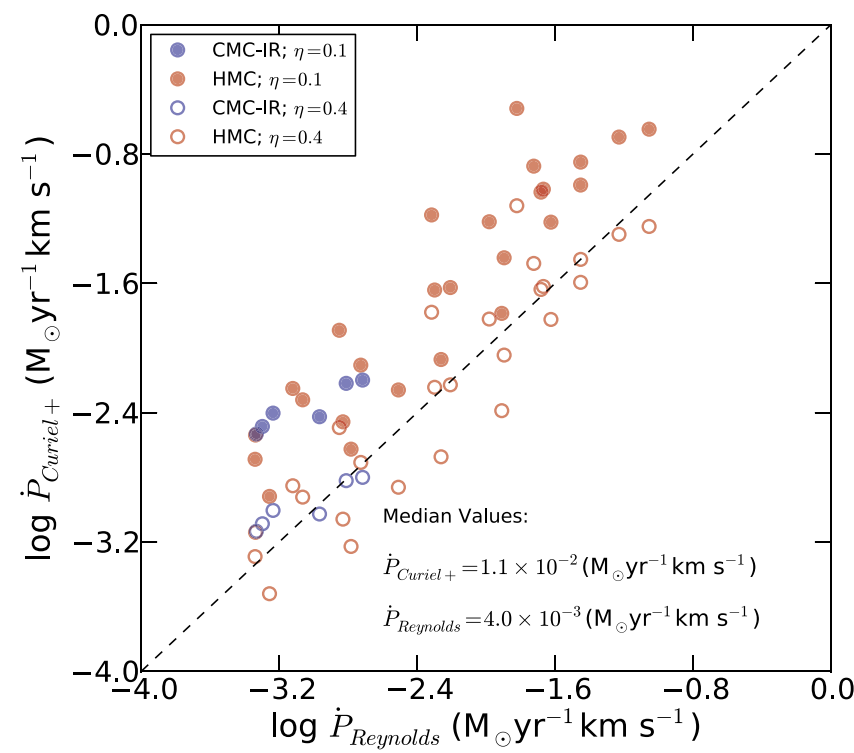

Figure 17. Momentum rate of an ionized jet from a shock of a neutral wind against the surrounding high density envelope as modeled Curiel et al. (1987) vs. the momentum rate of a partially optically thick ionized jet as modeled by Reynolds (1986) at $\nu=4.9 \mathrm{GHz}$. The colored dots and the empty circles represent the momentum rate calculated from shock ionization assuming a shock efficiency of $\eta=0.1$ and 0.4 , respectively. The dashed line represents the case where the momentum rate of the jet is sufficient to ionize itself.

toward our HMCs and CMC-IRs, respectively, with $0.2 \leqslant$ $\alpha \leqslant 1.2$ and for those sources that $\dot{P}_{\text {outflow }}$ is available in the literature. The dashed line represents the case where the momentum rate of the molecular outflow and the momentum

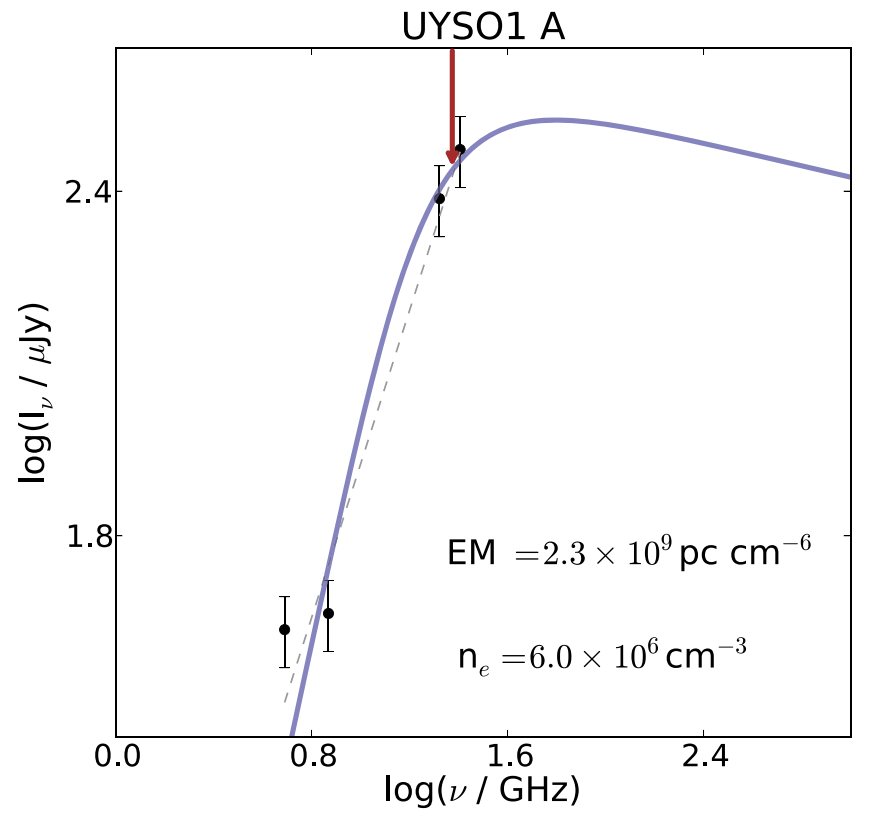

Figure 18. Error bars are an assumed uncertainty of $10 \%$ from the flux densities added in quadrature with an assumed $10 \%$ error in calibration. The continuous-blue line is the $\mathrm{H}$ II region fit using a spherical distribution. The dashed line is the best fit to the data from a power law of the form $S_{\nu} \propto \nu^{\alpha}$. The complete figure set (36 images) is available in the online journal.

(The complete figure set (36 images) is available.)

rate of the ionized jet are the same. We noticed that the momentum rate of the molecular outflow and the momentum rate of the associated ionized jet as estimated using the model of Reynolds (1986) is significantly similar for sources with $L_{\mathrm{bol}} \gtrsim 100 L_{\odot}$.

Figure 17 shows the momentum rate of the ionized jet as estimated from shock-induced ionization Curiel et al. (1987), assuming that it is the same as the momentum rate of the molecular outflow versus the momentum rate of a partially optically thick ionized jet as estimated using the model from Reynolds (1986). On average, we found that the required momentum rate in the jet as estimated from shock ionization is larger than the momentum rate calculated from a collimated jet as derived by Reynolds (1986) assuming a shock efficiency $\eta=0.1$ (colored dots in Figure 17). However, if the shock efficiency $\eta$ is increased to $\gtrsim 30 \%$ or if the jet velocities are $\sim 1600 \mathrm{~km} \mathrm{~s}^{-1}$, then the momentum rate of the jet would be sufficient to ionize itself (for example, the empty circles in Figure 17 represent the momentum rate from ionized jets assuming $\eta=0.4)$. For the very extreme cases where the momentum rate calculated from shock ionization is larger than the momentum rate calculated from Reynolds' model by a factor $\gtrsim 10$ and the velocities are $\sim 700 \mathrm{~km} \mathrm{~s}^{-1}$, the required shock efficiency would be required to be near unity.

\section{Appendix C Spectra of the Compact Radio Sources with Rising Spectral Index}

Figure 18 shows the fits for all 36 compact radio sources with rising spectral index detected in our survey as described in Section 2 (Compact Sources). 


\section{ORCID iDs}

V. Rosero (1) https://orcid.org/0000-0001-8596-1756

S. Kurtz (i) https://orcid.org/0000-0003-4444-5602

R. Cesaroni (1) https://orcid.org/0000-0002-2430-5103

E. D. Araya (1) https://orcid.org/0000-0001-6755-9106

K. M. Menten (1) https://orcid.org/0000-0001-6459-0669

L. Loinard (1) https://orcid.org/0000-0002-5635-3345

S. P. Ellingsen (10 https://orcid.org/0000-0002-1363-5457

S. Molinari 1 i https://orcid.org/0000-0002-9826-7525

\section{References}

AMI Consortium, Scaife, A. M. M., Hatchell, J., et al. 2011, MNRAS, 415,893

AMI Consortium, Scaife, A. M. M., Hatchell, J., et al. 2012, MNRAS, 420, 1019

Anglada, G. 1995, RMxAC, 27, 1, 67

Anglada, G. 1996, in ASP Conf. Ser. 93, Radio Emission from the Stars and the Sun, ed. A. R. Taylor \& J. M. Paredes (San Francisco, CA: ASP), 3

Anglada, G., Rodríguez, L. F., \& Carrasco-Gonzalez, C. 2015, in Advancing Astrophysics with the Square Kilometre Array (AASKA14) (Trieste: SISSA), 121

Anglada, G., Rodríguez, L. F., \& Carrasco-González, C. 2018, A\&ARv, 26, 3

Anglada, G., Villuendas, E., Estalella, R., et al. 1998, AJ, 116, 2953

Araya, E., Hofner, P., Sewiło, M., et al. 2007, ApJ, 669, 1050

Araya, E. D., Hofner, P., Goss, W. M., et al. 2008, ApJS, 178, 330

Bally, J., \& Zinnecker, H. 2005, AJ, 129, 2281

Beltrán, M. T., \& de Wit, W. J. 2016, A\&ARv, 24, 6

Beuther, H., Hunter, T. R., Zhang, Q., et al. 2004a, ApJL, 616, L23

Beuther, H., Schilke, P., \& Gueth, F. 2004b, ApJ, 608, 330

Beuther, H., Schilke, P., Menten, K. M., et al. 2002a, ApJ, 566, 945

Beuther, H., Schilke, P., Sridharan, T. K., et al. 2002b, A\&A, 383, 892

Beuther, H., Sridharan, T. K., \& Saito, M. 2005, ApJL, 634, L185

Beuther, H., Vlemmings, W. H. T., Rao, R., \& van der Tak, F. F. S. 2010, ApJL, 724, L113

Beuther, H., Walsh, A., Schilke, P., et al. 2002c, A\&A, 390, 289

Beuther, H., Zhang, Q., Sridharan, T. K., Lee, C.-F., \& Zapata, L. A. 2006, A\&A, 454, 221

Bonnell, I. A., Bate, M. R., \& Zinnecker, H. 1998, MNRAS, 298, 93

Caratti o Garatti, A., Stecklum, B., Linz, H., Garcia Lopez, R., \& Sanna, A. 2015, A\&A, 573, A82

Carey, S. J., Noriega-Crespo, A., Price, S. D., et al. 2005, BAAS, 37, 1252

Carral, P., Kurtz, S., Rodríguez, L. F., et al. 1999, RMxAA, 35, 97

Carrasco-González, C., Rodríguez, L. F., Anglada, G., et al. 2010, Sci, 330, 1209

Cesaroni, R., Sánchez-Monge, Á., Beltrán, M. T., et al. 2016, A\&A, 588, L5

Cesaroni, R., Felli, M., Jenness, T., et al. 1999, A\&A, 345, 949

Cesaroni, R., Massi, F., Arcidiacono, C., et al. 2013, A\&A, 549, A146

Cesaroni, R., Moscadelli, L., Neri, R., et al. 2018, A\&A, 612, A103

Cesaroni, R., Neri, R., Olmi, L., et al. 2005, A\&A, 434, 1039

Cesaroni, R., Pestalozzi, M., Beltrán, M. T., et al. 2015, A\&A, 579, A71

Cooper, H. D. B., Lumsden, S. L., Oudmaijer, R. D., et al. 2013, MNRAS, 430, 1125

Curiel, S., Canto, J., \& Rodriguez, L. F. 1987, RMxAA, 14, 595

Curiel, S., Ho, P. T. P., Patel, N. A., et al. 2006, ApJ, 638, 878

Curiel, S., Rodriguez, L. F., Bohigas, J., et al. 1989, ApL\&C, 27, 299

Cyganowski, C. J., Brogan, C. L., Hunter, T. R., Churchwell, E., \& Zhang, Q. 2011, ApJ, 729, 124

Davis, C. J., Varricatt, W. P., Todd, S. P., \& Ramsay Howat, S. K. 2004, A\&A, 425,981

de Pree, C. G., Rodriguez, L. F., \& Goss, W. M. 1995, RMxAA, 31, 39

Dyson, J. E., \& Williams, D. A. 1980, Physics of the Interstellar Medium (New York: Halsted Press)

Dzib, S. A., Loinard, L., Mioduszewski, A. J., et al. 2013, ApJ, 775, 63

Dzib, S. A., Loinard, L., Rodríguez, L. F., et al. 2015, ApJ, 801, 91

Ellsworth-Bowers, T. P., Rosolowsky, E., Glenn, J., et al. 2015, ApJ, 799, 29

Fallscheer, C., Beuther, H., Sauter, J., Wolf, S., \& Zhang, Q. 2011, ApJ, 729, 66

Fallscheer, C., Beuther, H., Zhang, Q., Keto, E., \& Sridharan, T. K. 2009, A\&A, 504, 127

Forbrich, J., Schreyer, K., Posselt, B., Klein, R., \& Henning, T. 2004, ApJ, 602,843
Franco, J., García-Segura, G., Kurtz, S. E., \& Arthur, S. J. 2007, ApJ, 660, 1296

Garay, G., Brooks, K. J., Mardones, D., \& Norris, R. P. 2003, ApJ, 587, 739 Garay, G., \& Rodriguez, L. F. 1990, ApJ, 362, 191

Goddi, C., Zhang, Q., \& Moscadelli, L. 2015, A\&A, 573, A108

Guzmán, A. E., Garay, G., Brooks, K. J., \& Voronkov, M. A. 2012, ApJ, 753,51

Hoare, M. G., Kurtz, S. E., Lizano, S., Keto, E., \& Hofner, P. 2007, Protostars and Planets V (Tucson, AZ: Univ. of Arizona Press), 181

Hofner, P., Cesaroni, R., Kurtz, S., et al. 2017, ApJ, 843, 99

Hofner, P., Cesaroni, R., Olmi, L., et al. 2007, A\&A, 465, 197

Hofner, P., Kurtz, S., Ellingsen, S. P., et al. 2011, ApJL, 739, L17

Hofner, P., Wyrowski, F., Walmsley, C. M., \& Churchwell, E. 2000, ApJ, 536, 393

Johnston, K. G., Shepherd, D. S., Robitaille, T. P., \& Wood, K. 2013, A\&A, 551, A43

Keto, E. 2002, ApJ, 568, 754

Keto, E. 2003, ApJ, 599, 1196

Keto, E. 2007, ApJ, 666, 976

Konigl, A., \& Pudritz, R. E. 2000, in Protostars and Planets IV, ed. V. Mannings (Tucson, AZ: Univ. Arizona Press), 759

Kounkel, M., Hartmann, L., Loinard, L., et al. 2014, ApJ, 790, 49

Kurtz, S. 2005, in IAU Symp. 227, Massive Star Birth: A Crossroads of Astrophysics, ed. R. Cesaroni (Cambridge: Cambridge Univ. Press), 111

Kurtz, S., Churchwell, E., \& Wood, D. O. S. 1994, ApJS, 91, 659

Lawrence, A., Warren, S. J., Almaini, O., et al. 2007, MNRAS, 379, 1599

Lee, H.-T., Liao, W.-T., Froebrich, D., et al. 2013, ApJS, 208, 23

Lee, H.-T., Takami, M., Duan, H.-Y., et al. 2012, ApJS, 200, 2

López-Sepulcre, A., Cesaroni, R., \& Walmsley, C. M. 2010, A\&A, 517, A66

Marti, J., Rodriguez, L. F., \& Reipurth, B. 1995, ApJ, 449, 184

Martí, J., Rodríguez, L. F., \& Reipurth, B. 1998, ApJ, 502, 337

McMullin, J. P., Waters, B., Schiebel, D., Young, W., \& Golap, K. 2007, in ASP Conf. Ser. 376, Astronomical Data Analysis Software and Systems XVI, ed. R. A. Shaw, F. Hill, \& D. J. Bell (San Francisco, CA: ASP), 127

Molinari, S., Brand, J., Cesaroni, R., Palla, F., \& Palumbo, G. G. C. 1998, A\&A, 336, 339

Molinari, S., Swinyard, B., Bally, J., et al. 2010, PASP, 122, 314

Moscadelli, L., Cesaroni, R., Sánchez-Monge, Á., et al. 2013, A\&A, 558, A145

Moscadelli, L., Sánchez-Monge, Á., Goddi, C., et al. 2016, A\&A, 585, A71

Motte, F., Bontemps, S., \& Louvet, F. 2018, ARA\&A, 56, 41

Navarete, F., Damineli, A., Barbosa, C. L., \& Blum, R. D. 2015, MNRAS, 450,4364

Olnon, F. M. 1975, A\&A, 39, 217

Ortiz-León, G. N., Loinard, L., Mioduszewski, A. J., et al. 2015, ApJ, 805, 9

Palau, A., Estalella, R., Girart, J. M., et al. 2007a, A\&A, 465, 219

Palau, A., Estalella, R., Ho, P. T. P., Beuther, H., \& Beltrán, M. T. 2007b, A\&A, 474, 911

Panagia, N. 1973, AJ, 78, 929

Panagia, N., \& Felli, M. 1975, A\&A, 39, 1

Pech, G., Loinard, L., Dzib, S. A., et al. 2016, ApJ, 818, 116

Pilbratt, G. L., Riedinger, J. R., Passvogel, T., et al. 2010, A\&A, 518, L1

Purcell, C. R., Hoare, M. G., Cotton, W. D., et al. 2013, ApJS, 205, 1

Purser, S. J. D., Lumsden, S. L., Hoare, M. G., et al. 2016, MNRAS, 460, 1039

Rathborne, J. M., Jackson, J. M., Chambers, E. T., et al. 2010, ApJ, 715, 310

Rathborne, J. M., Jackson, J. M., \& Simon, R. 2006, ApJ, 641, 389

Reynolds, S. P. 1986, ApJ, 304, 713

Rieke, G. H., Young, E. T., Engelbracht, C. W., et al. 2004, ApJS, 154, 25

Rivilla, V. M., Martín-Pintado, J., Jiménez-Serra, I., \& Rodríguez-Franco, A. 2013, A\&A, 554, A48

Robitaille, T., \& Bressert, E. 2012, APLpy: Astronomical Plotting Library in Python, Astrophysics Source Code Library, ascl:1208.017

Rodriguez, L. F., Garay, G., Curiel, S., et al. 1994, ApJL, 430, L65

Rodríguez, L. F., González, R. F., Montes, G., et al. 2012, ApJ, 755, 152

Rodríguez, L. F., Moran, J. M., Franco-Hernández, R., et al. 2008, AJ, 135,2370

Rosero, V., Hofner, P., Claussen, M., et al. 2016, ApJS, 227, 25

Rosero, V., Hofner, P., McCoy, M., et al. 2014, ApJ, 796, 130

Sánchez-Monge, Á., Beltrán, M. T., Cesaroni, R., et al. 2013a, A\&A, 550, A21

Sánchez-Monge, Á., Kurtz, S., Palau, A., et al. 2013b, ApJ, 766, 114

Sánchez-Monge, Á., López-Sepulcre, A., Cesaroni, R., et al. 2013c, A\&A, 557, A94

Sanna, A., Kölligan, A., Moscadelli, L., et al. 2019a, A\&A, 623, A77

Sanna, A., Moscadelli, L., Cesaroni, R., et al. 2016, A\&A, 596, L2

Sanna, A., Moscadelli, L., Goddi, C., et al. 2019b, A\&A, 623, L3 
Sanna, A., Moscadelli, L., Goddi, C., Krishnan, V., \& Massi, F. 2018, A\&A, 619, A107

Shepherd, D. S., \& Churchwell, E. 1996, ApJ, 457, 267

Shepherd, D. S., Nürnberger, D. E. A., \& Bronfman, L. 2004, ApJ, 602, 850

Shepherd, D. S., Povich, M. S., Whitney, B. A., et al. 2007, ApJ, 669, 464

Shepherd, D. S., Yu, K. C., Bally, J., \& Testi, L. 2000, ApJ, 535, 833

Shu, F. H., Adams, F. C., \& Lizano, S. 1987, ARA\&A, 25, 23

Shu, F. H., Lizano, S., Ruden, S. P., \& Najita, J. 1988, ApJL, 328, L19

Sicilia-Aguilar, A., Hartmann, L. W., Szentgyorgyi, A. H., et al. 2005, AJ, 129,363

Sridharan, T. K., Beuther, H., Schilke, P., Menten, K. M., \& Wyrowski, F. 2002, ApJ, 566, 931

Su, Y.-N., Liu, S.-Y., Chen, H.-R., Zhang, Q., \& Cesaroni, R. 2007, ApJ, 671,571

Tan, J. C., Beltrán, M. T., Caselli, P., et al. 2014, Protostars and Planets VI (Tucson, AZ: Univ. Arizona Press), 149

Tan, J. C., Kong, S., Zhang, Y., et al. 2016, ApJL, 821, L3

Tanaka, K. E. I., Tan, J. C., \& Zhang, Y. 2016, ApJ, 818, 52

Tobin, J. J., Hartmann, L., Furesz, G., Mateo, M., \& Megeath, S. T. 2009, ApJ, 697,1103
Urquhart, J. S., Csengeri, T., Wyrowski, F., et al. 2014, A\&A, 568, A41 van Buren, D., Mac Low, M.-M., Wood, D. O. S., \& Churchwell, E. 1990 ApJ, 353, 570

Varricatt, W. P., Davis, C. J., Ramsay, S., \& Todd, S. P. 2010, MNRAS, 404, 661

Varricatt, W. P., Thomas, H. S., Davis, C. J., Ramsay, S., \& Currie, M. J. 2013 A\&A, 554, A9

Walmsley, M. 1995, RMxAC, 1, 137

Wang, K., Zhang, Q., Testi, L., et al. 2014, MNRAS, 439, 3275

Wilner, D. J., De Pree, C. G., Welch, W. J., \& Goss, W. M. 2001, ApJL, 550, L81

Wolf-Chase, G., Arvidsson, K., \& Smutko, M. 2017, ApJ, 844, 38

Wood, D. O. S., \& Churchwell, E. 1989a, ApJ, 340, 265

Wood, D. O. S., \& Churchwell, E. 1989b, ApJS, 69, 831

Xie, T., Mundy, L. G., Vogel, S. N., \& Hofner, P. 1996, ApJL, 473, L131

Zapata, L. A., Rodríguez, L. F., Ho, P. T. P., Beuther, H., \& Zhang, Q. 2006, AJ, 131, 939

Zhang, Q., Sridharan, T. K., Hunter, T. R., et al. 2007, A\&A, 470, 269

Zhang, Y., Tan, J. C., \& Hosokawa, T. 2014, ApJ, 788, 166 